\title{
Ion-shift reagent binding energy and the shift-mass correlation in ion mobility spectrometry
}

\author{
Roberto Fernandez-Maestre, ${ }^{\mathrm{a}, \mathrm{b}}$ Mahmoud Tabrizchi, ${ }^{\mathrm{c}}$ and Dairo Meza-Morelos ${ }^{\mathrm{a}}$ \\ ${ }^{\text {a } U n i v e r s i d a d ~ d e ~ C a r t a g e n a, ~ C a m p u s ~ d e ~ S a n ~ P a b l o, ~ P r o g r a m a ~ d e ~ Q u i ́ m i c a, ~ C a r t a g e n a, ~ C o l o m b i a ~}$ \\ *dmezam@unicartagena.edu.co \\ ${ }^{\mathrm{b}}$ National Research Nuclear University MEPhI (Moscow Engineering Physics Institute). Kashirskoe sh. 31, \\ Moscow, 115409, Russia \\ ${ }^{c}$ Department of Chemistry, Isfahan University of Technology, Isfahan 84156-83111, Iran
}

\begin{abstract}
Ion mobility spectrometry is widely used for the detection of illegal substances and explosives in airports, ports, custom, some stations and many other important places. This task is usually complicated by false positives caused by overlapping the target peaks with that of interferents, commonly associated with samples of interest. Shift reagents (SR) are species that selectively change ion mobilities through adduction with analyte ions when they are introduced in IMS instruments. This characteristic can be used to discriminate false positives because the interferents and illegal substances respond differently to SR depending on the structure and size of analytes and their interaction energy with SR. This study demonstrates that ion mobility shifts upon introduction of SR depend, not only on the ion masses, but on the interaction energies of the ion:SR adducts. In this study, we introduced five different SRs using ESI-IMS-MS to study the effect of the interaction energy and size on mobility shifts. The mobility shifts showed a decreasing trend as the molecular weight increased for the series of compounds ethanolamine, valinol, serine, threonine, phenylalanine, tyrosine, tributylamine, tryptophan, desipramine, and tribenzylamine. It was proved that the decreasing trend was partially due to the inverse relation between the mobility and drift time and hence, the shift in drift time better reflects the pure effect of SR on the mobility of analytes. Yet the drift time shift exhibited a mild decrease with the mass of ions. Valinol pulled out from this trend because it had a low binding energy interaction with all the SR and, consequently, its clusters were short-lived. This short lifetime produced fewer collisions against the buffer gas and a drift time shorter compared to those of ions of similar molecular weight. Analyte ion:SR interactions were calculated using Gaussian. IMS with the introduction of SR could be the choice for the free-interferents detection of illegal drugs, explosives, and biological and warfare agents. The suppression of false positives could facilitate the transit of passengers and cargos, rise the confiscation of illicit substances, and save money and distresses due to needless delays.
\end{abstract}

Keywords: Adduction, ion mobility spectrometry, mass spectrometry, shift reagent, valinol, buffer gas modifier

\section{INTRODUCTION}

Ion mobility spectrometry is an analytical separation technique introduced in 1970 by Francis Karasek (Cohen and Karasek 1970). IMS separates ions according to their size to charge ratio under the acceleration of an electric field, while collisions with the buffer gas decelerate the ions (Mason and McDaniel 1988). The collision cross-section, CCS, of the ions is proportional to the time the ions spend to reach the detector. IMS is the instrumental technique most used to detect prohibited substances such as explosives, chemical and biological warfare agents, and drugs in airports, ports, customs (Eiceman and Stone 2004), and prisons (Vautz et al. 2009). False positives caused by interfering ions that overlap with the searched ions are annoying incidents for transporters and passengers. The introduction of shift reagents (SR) in the buffer gas of drifttube ion mobility spectrometers has been used to selectively change ion mobilities and separate analytes that overlap in IMS by adduction with analyte ions. Hence, using SR may decrease the number of false positives by selectively changing ion mobilities and would save time to customs officials, transportation personnel, and passengers. Shift reagents can be considered as a deliberate impurity added to the buffer gas that may react with the ions travelling to lengthen their drift time. Eiceman et al. (1993) eliminated the ammonia interference using ketones as SR to form clusters of hydrazine and monomethylhydrazine; after clustering with ketones, the drift times of these species changed selectively, hence, separating them. Bollan et al. (2007) introduced ketones into the buffer gas to form complexes with hydrazines avoiding ammonia interference. In 2014, overlapping picoline isomers were separated using 2-butanol due to the formation of different nanocluster product ions with different cross-section areas. These cross-sections depended on the position of the methyl group on the pyridine ring (Ghaemi and Alizadeh 2014). The effect of the introduction of polar SR in the 
buffer gas has been studied and electrostatic attraction, energetically possible conformations, hydrogenbonding, and steric repulsion were proposed as the origin of mobility shifts (Levin et al. 2007). Roscioli et al. (2014) showed that analogous proton affinities of the SR were significant to obtain substantial mobility shifts. The main origin of mobility shifts after the introduction of SR in IMS is the cluster binding energy and the steric hindrance on the charge site instead of size. This was demonstrated by Campbell et al (2014). Tsai et al. (2016) added SR to produce large clusters of ammonium nitrate and urea nitrate, commonly used as improvised explosive devices, to overcome challenges for the detection of these species such as their low mass. Butcher et al. (2019) added solution additives and gas-phase SR to heme proteins and found changes in the mobility profiles depending on the size of the SR used (methanol, acetone, or acetonitrile). They attributed the changes in mobility to clustering and considered that these experiments open new avenues for the manipulation and interrogation of biomolecules in the gas phase. Similar results were obtained for methanol, acetonitrile, and acetone as SR in the analysis of growth hormone-releasing hormone using trapped ion mobility-mass spectrometry (Fouque et al. 2019). Parchami et al. (2017a) eliminated peak overlap in the analysis of biogenic amines (histamine, putrescine, cadaverine, and tyramine) in canned fish samples by using 8-crown-6 as SR in the carrier gas. We have used SR to analyze valinol and other analytes introducing several SR (Fernandez-Maestre 2018).

The formation of larger and slower SR-ion adducts than its original size and speed, decreases the mobility of ions upon introduction of SR in the drift gas in IMS. Several parameters are used to explain these changes in mobility: proton affinity and size, inductive effects, SR-ion binding energy, ion charge delocalization, steric hindrance, and number of adduction sites (Rawat et al. 2015). Inductive effects strengthen or weaken ion:SR interactions and the steric hindrance on the charge produced by bulky substituents shields the ion charge from the adduction of SR molecules and delocalizes the charge weakening the ion:SR interactions. Because IMS separates ions based on the size to charge ratio, and the clustering of ions to SR yields larger-sized adducts, it increases the collisions against the molecules of the buffer gas, and resulting in a greater mobility shift. Therefore, larger mobility shifts are obtained with bulkier SR. However, the larger the SR the smaller the number of SR molecules clustering with a certain ion. Proton affinities, intramolecular hydrogen bonds, and steric and inductive effects, are considered when computing the interaction energies of analyte-SR and, consequently, the most used factors to explain mobility changes induced by SR are the number of adduction sites, interaction energies, and SR size (Fernandez-Maestre 2018). Oberreit et al (2015) applied differential mobility-mass spectrometry to observe the sorption of 1-13 vapor molecules onto iodide cluster ions in air at $300 \mathrm{~K}$. Measured CCS shifts were compared to predictions based on the KelvinThomson-Raoult (KTR) and Langmuir adsorption models. They found that the models fit very well to measurements, but the earliest stages of vapor uptake were not well described by the KTR model. The introduction of SR in the buffer gas in IMS has been reviewed (Puton et al. 2008; Waraksa et al. 2016).

The mobility shifts of valinol after the introduction of several buffer gas SR have been studied. The following percent changes in mobilities have been obtained:- 5.1\% (trifluoromethyl benzyl alcohol), -18\% (methyl-2chloro propionate), $-7.1 \%$ (water) $-28 \%$ (ethyl lactate), $-9.8 \%$ (2-butanol), and -21\% (nitrobenzene) after introducing 2.3, 1.0, 879, 1.7, 6.8, and $1.0 \mathrm{mmol} \mathrm{m}^{-3}$ of the SR in the buffer gas, respectively (FernandezMaestre et al. 2010a, 2010b). In the same conditions, serine, a compound with a similar mass and structure showed larger mobility shifts than valinol. As a result, valinol moved away from the mobility shift-mass correlation lines. In this study, we used IMS and theoretical calculations to elucidate the origin of the behavior of valinol in the mobility shift-mass correlations after SR introduction. These mobility shift-mass correlations and the explanation of the departure of some ions from these correlations have not been reported before. This is important to explain this chemical behavior to take a step ahead in the study and design of SR for the IMS detection of illegal substances.

\section{EXPERIMENTAL}

\subsection{Instrument}

The methodology has been modified from that described elsewhere and only a summary is given here (Wittmer et al. 1994). An electrospray-ionization (ESI) atmospheric-pressure ion mobility spectrometer coupled to a quadrupole mass spectrometer was used in these experiments (Figure S1). Routine operating parameters for this instrument were: sample flow, $3 \mu \mathrm{min}^{-1}$; ESI voltage, $15.6 \mathrm{kV}$; voltage at the first ring, $12.1 \mathrm{kV}$; voltage at the gate, $10.80 \pm 0.01 \mathrm{kV}$; gate closure potential, $\pm 40 \mathrm{~V}$; gate pulse width, $0.1 \mathrm{~ms}$; scan time, $35 \mathrm{~ms}$; number of averages per spectrum, 100-1000; pressure, 688-711 Torr (in Pullman, WA, USA); nitrogen flow, 0.93 liter $\mathrm{min}^{-1}$; drift tube temperature, $100-250 \pm 2{ }^{\circ} \mathrm{C}$. The ABB Extrel mass spectrometer (Pittsburgh, PA, USA, 0-4000 amu) was operated in three modes. worked in single ion monitoring ion 
mobility spectrometry (SIM-IMS), radiofrequency-only ion mobility spectrometry (IMS), and mass spectrometry (MS) modes. In SIM-IMS mode, only the ion mobility spectrum of ions of a given mass to charge ratio or a range of masses is obtained. In IMS mode, the total ion mobility spectrum is obtained; and in MS mode, mass spectra are obtained.

The ion mobility spectrometer was made at Washington State University, WSU, (WA, USA) and experiments were performed there. It comprised an electrospray ionization source, a $25-\mathrm{cm}$ drift tube coupled, and a quadrupole mass spectrometer detector. The reaction region length was $7.5 \mathrm{~cm}$. A Bradbury-Nielsen ion gate separated the desolvation and drift regions; a countercurrent of dry, preheated $\mathrm{N}_{2}$ buffer gas was introduced through the end of the drift tube.

\subsection{Reagents}

Desipramine, ethanolamine, methionine, phenylalanine, serine, threonine, tributylamine, tribenzylamine, tryptophan, tyrosine, and valinol were used as analytes; 2,4-lutidine and 2,6-di-tert-butyl pyridine (DTBP) as chemical standards; and 2-butanol, ethyl lactate, methyl-2-chloro propionate, trifluoromethyl benzyl alcohol, and water as SR. These reagents plus methanol, water, and acetic acid (ACS reagent grade, $\geq 97$ or $98 \%$ purity) were purchased from Sigma Aldrich Chemical Co. (Milwaukee, WI, USA). The structures of compounds used in this study are shown in Figure S2. These analytes were selected because their different molecular weights and structures were required to compare the effects of size, interaction energy, and steric hindrance on the mobilities of these molecules with those of valinol with the introduction of SR into the buffer gas. The chemical standards, DTBP and 2,4-lutidine, were selected because they are commonly used as chemical standards (Eiceman et al. 2002).

\subsection{Buffer gas}

$\mathrm{N}_{2}$ was used as the buffer gas. The humidity of the buffer gas was an average of 10 ppmv. measured with a GE Moisture Image Series 1 Instrument (Billerica, MA, USA), (Fernandez-Maestre et al. 2010a). The drift tube was heated at $150^{\circ} \mathrm{C}$ using 5 - and $10-\mathrm{cm} 3 / 8^{\prime \prime}$ firerod cartridge heaters (Watlow, Anaheim CA, USA). We waited until the drift tube was saturated with the SR to start drift time measurements so that the clusters were formed in the ion source. Saturation was considered to be reached when the mobilities of the analytes stabilized over time. The concentration of SR was set using the flow rate of liquid SR, the experimental conditions, and the equation of state.

\subsection{Solutions preparation and injection}

Solutions were prepared at $50-\mu \mathrm{M}$ (analytes) or $10-\mu \mathrm{M}$ (chemical standards) concentrations in ESI solution (47.5\% methanol: $47.5 \%$ water: $5 \%$ acetic acid). Liquid samples or blank solutions (ESI solution) were electrosprayed continuously into the drift tube. Liquid SR were introduced as vapors into the buffer gas line before the buffer gas heater through a heated cross-junction.

\subsection{Computational-Theoretical Studies}

The Gaussian 09 program (Revision D.01) (Frisch et al. 2009) was used for the theoretical-computational calculations at $150^{\circ} \mathrm{C}$ using the X3LYP-GD3/6-311++(d,p) functional, which includes the Grimme scatter correction. Also, a new, larger, ultrafine grid was used, useful when high precision is desired. Geometry optimization was performed for the analytes, the SR, their protonated species, and their adducts. The structures of the molecules were then used as starting points to determine the geometries and energies. The interaction energies (IE) were calculated using the equation $I E=E_{\text {adduct }}-\left(E_{S R}+E_{\text {analyte ion }}\right)$. The proton affinities were extracted from the calculations performed on the protonated analytes (Foresman and Frisch 1996). Gibbs free energies $(\Delta \mathrm{G})$, enthalpies $(\Delta \mathrm{H})$ and entropies, reported as $\mathrm{T} \Delta \mathrm{S}$, were calculated for the complex formation reactions studied.

\subsection{Identification of peaks in the spectra}

The identification of analytes was performed by comparing their $m / z$ signal in mass spectrometry to the molecular weight of their protonated molecules or clusters. Also, analyte peaks and their clusters in the IMS spectrum were analyzed by SIM-IMS for identification. Additionally, the reduced mobilities of the protonated analytes were compared with values from the literature. 


\subsection{Instrument calibration}

Under given conditions, the product of the reduced mobility of an ion, $K_{0}$, times its drift time is constant. This lets the reduced mobility to be calculated from that of a calibrant, $K_{0, c}$, the calibrant drift time, $c_{t}$, and the analyte drift time at the same conditions, $d_{t}$ :

2,6-di-tert-butylpyridine was used as a calibrant.

$$
K_{0}=K_{0, c} \frac{c_{t}}{d_{t}}(1)
$$

\subsection{Experiments}

For every analyte ion and shift reagent we tried five SR concentrations (for a total 210 experiments) but only 48 at the highest concentrations were reported because the others showed a similar trend.

\section{RESULTS AND DISCUSSIONS}

The number of reduced mobility measurements was $>3$. Reproducibilities of 0.3 to $0.6 \%$ were obtained for reduced mobilities. The raw database can be found in Fernandez-Maestre (2017). The mass spectrometer had a low resolution, but this was not a constraint because the peaks of interest were easily differentiated.

\subsection{Spectra of the buffer gas}

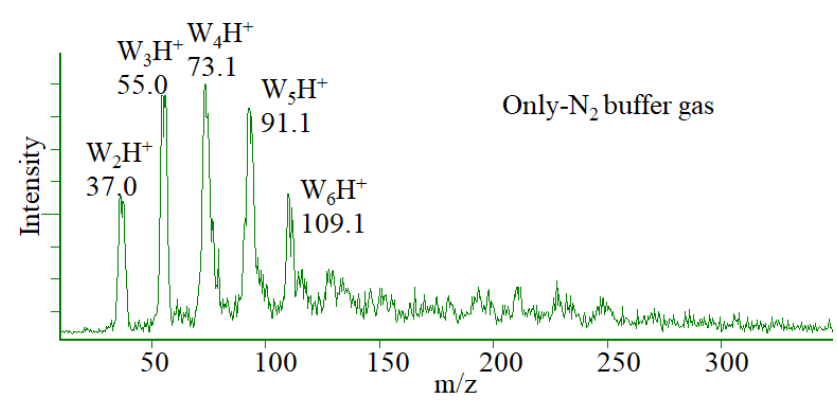

Fig. 1 Mass spectrum of the electrospray solution in pure $\mathrm{N}_{2}$ buffer gas at $150^{\circ} \mathrm{C}$. Clusters with up to six water molecules are shown at $m / z$ 37.0, 55.0, 73.1,91.1, and 109.1. W: water

Figure 1 shows the MS spectrum of the ESI solvent before introducing SR in the buffer gas. Water clusters with two to six molecules are seen at $\mathrm{m} / \mathrm{z} 37.0,55.0,73.1,91.1$, and 109.1. The spectrum looks clean with only noise after $m / z 109.1$.

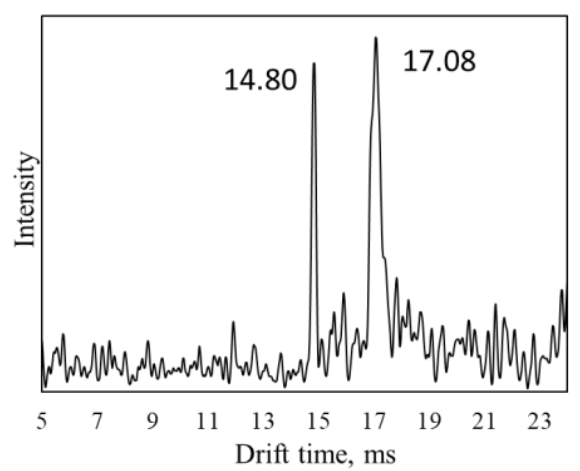

Fig. 2 Ion mobility spectrum of the electrospray solution in pure $\mathrm{N}_{2}$ buffer gas at $150^{\circ} \mathrm{C}$. The water clusters shown in Figure 1 coalesced into a single mobility peak at $17.08 \mathrm{~ms}$ due to the equilibria between these species. The peak at 14.80 may be due to clusters of ammonium ions with water.

Figure 2 shows the IMS spectrum of the ESI solvent before introducing any SR in the buffer gas. Both the IMS and mass spectra show no contamination: only water peaks are seen in the mass spectrum and only peaks from the components of the ESI solvent are seen in the IMS spectrum; all other peaks are small. The 
elimination of contamination is important to prevent the mobility shifts due to clustering of the analytes to contaminants. The peak at 14.80 may be due to clusters of ammonium contaminating ions with water because the ammonium peak always appears before the hydronium peak in IMS (Bahrami \& Tabrizchi, 2012). The difference between ammonium and water clusters with the same $n$ number is only one $\mathrm{m} / \mathrm{z}$ unit. For example, water clusters appear at $\mathrm{m} / \mathrm{z} 37,55,73,91,109$ while the ammonium clusters appear at $\mathrm{m} / \mathrm{z} 38,56,74,92$, 110. Because the mass spectrometer was low resolution, the two sets of clusters were not separated in the mass spectrum. All hydronium water clusters in the IMS spectrum merged into one single mobility peak at $17.08 \mathrm{~ms}$, with a weighted average of the ion mobilities of all water ions. Due to the following equilibria, they quickly interconvert into each other many times during their travel through the drift tube,

$$
\left(\mathrm{H}_{2} \mathrm{O}\right)_{\mathrm{a}} \mathrm{H}^{+} \leftrightarrow\left(\mathrm{H}_{2} \mathrm{O}\right)_{\mathrm{a}-\mathrm{y}} \mathrm{H}^{+}+\mathrm{yH}_{2} \mathrm{O}
$$

This is also true for the peak at $14.80 \mathrm{~ms}$ that is the result of all ammonium water clusters.

\subsection{Spectra of valinol in pure $N_{2}$ buffer gas or when the buffer gas was doped with 2-butanol}

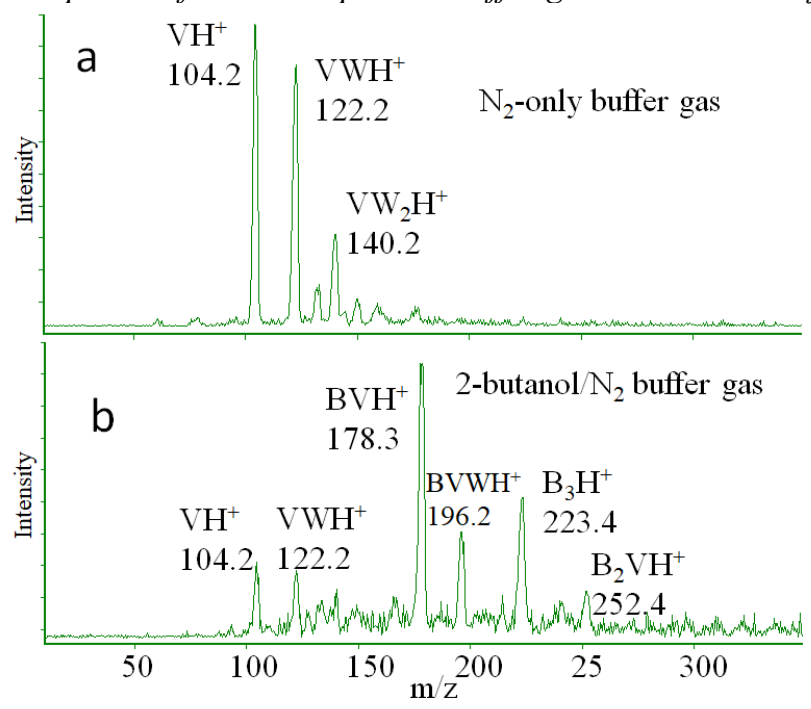

Fig. 3 Mass spectra of a 100- $\mu \mathrm{M}$ solution of valinol in nitrogen-only buffer gas (a) and when $0.68 \mathrm{mmol} \mathrm{m}^{-3}$ of 2-butanol (b) was introduced into the buffer gas. Protonated valinol ion $\left(\mathrm{VH}^{+}\right)$, hydrated valinol cluster $\left(\mathrm{VWH}^{+}\right)$, doubly hydrated valinol cluster $\left(\mathrm{VW}_{2} \mathrm{H}^{+}\right)$, 2-butanol:valinol cluster $\left(\mathrm{BVH}^{+}\right)$, hydrated 2butanol:valinol cluster $\left(\mathrm{BVWH}^{+}\right)$, 2-butanol trimer $\left(\mathrm{B}_{3} \mathrm{H}^{+}\right)$, and cluster of valinol with two 2-butanol molecules $\left(\mathrm{B}_{2} \mathrm{VH}^{+}\right)$are seen

Figure 3 shows the mass spectra of valinol solutions in nitrogen-only buffer gas (a) and when $0.68 \mathrm{mmol} \mathrm{m}^{-3}$ $(5.0 \mu \mathrm{L} / \mathrm{min}$ flow rate) of 2-butanol (b) was introduced into the buffer gas by the end of the drift tube together with the buffer gas. The protonated valinol ion $\left(\mathrm{VH}^{+}\right)$and its clusters with water (the hydrated valinol cluster, $\mathrm{VWH}^{+}$, and the doubly hydrated valinol cluster, $\mathrm{VW}_{2} \mathrm{H}^{+}$) are the dominant peaks without introducing 2butanol in the buffer gas. The water clusters seen in Figure 2 disappeared from the mass spectra by adduction with 2-butanol or the charge was stripped by valinol and 2-butanol due to the lower proton affinity of water. However, when 2-butanol was present in the buffer gas, the intensity of valinol ion decreased due to the displacement of the equilibria to the formation of clusters of valinol with 2-butanol, such as $\mathrm{BVWH}^{+}, \mathrm{BVH}^{+}$, $\mathrm{B}_{2} \mathrm{VH}^{+}$, and others of minor intensity. The peak intensity of the clusters was higher than that of protonated valinol even at a relatively low 2-butanol concentration $\left(0.68 \mathrm{mmol} \mathrm{m}^{-3}\right)$ indicating that 2-butanol effectively adducted to valinol without major interferences from steric hindrance and else. 


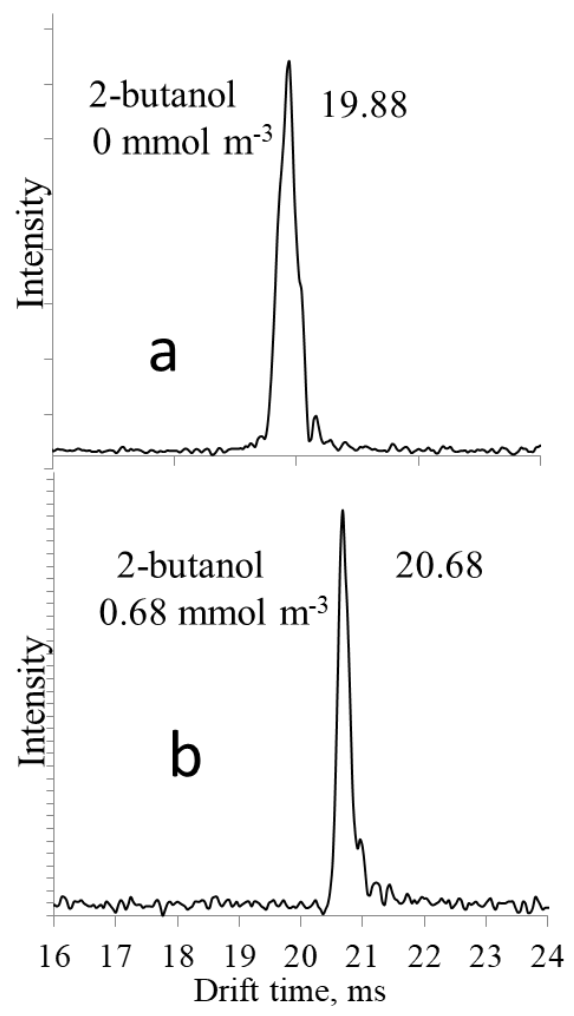

Fig. 4 Mobility spectra of a $100-\mu \mathrm{M}$ solution of valinol in $\mathrm{N}_{2}$-only buffer gas (a) and when $0.68 \mathrm{mmol} \mathrm{m}^{-3}$ of 2-butanol was introduced in the buffer gas. There was a $0.80 \mathrm{~ms}$ mobility shift when 2-butanol was present in the buffer gas

Figure 4a shows the ion mobility spectra of valinol in $\mathrm{N}_{2}$-only buffer gas; the valinol peak is observed at 19.88 $\mathrm{ms}$. When $0.68 \mathrm{mmol} \mathrm{m}^{-3}$ of 2-butanol was introduced in the buffer gas the valinol peak displaced to 20.68 ms. This 2-butanol concentration corresponded to a flow rate of $5 \mu \mathrm{L} / \mathrm{hr}$ of 2-butanol vaporized into the buffer gas. The drift time of valinol increased due to the occurrence of a hydrogen bond between the oxygen atom in 2-butanol and the charge on the amine group in valinol that stabilized the charge by sharing it with the hydroxyl group in 2-butanol. The valinol peak shifted from 19.88 to $20.68 \mathrm{~ms}$, a $0.80 \mathrm{~ms}$ mobility shift, according to a series of chain equilibria between the protonated valinol $\left(\mathrm{VH}^{+}\right)$and 2-butanol (B).

$$
\begin{gathered}
\mathrm{VH}^{+}+\mathrm{B} \leftrightarrow \mathrm{BVH}^{+} \\
\mathrm{BVH}^{+}+\mathrm{B} \leftrightarrow \mathrm{B}_{2} \mathrm{VH}^{+} \\
\mathrm{B}_{2} \mathrm{VH}^{+}+\mathrm{B} \leftrightarrow \mathrm{B}_{3} \mathrm{VH}^{+} \ldots \\
\mathrm{VH}_{3} \mathrm{O}^{+}+\mathrm{B} \leftrightarrow \mathrm{BVH}_{3} \mathrm{O}^{+} \ldots
\end{gathered}
$$

In fact, all ion- molecule reactions that may occur in the drift tube under influence of water (W) and a shift reagent $(\mathrm{SR})$ can be generally presented in Fig. 5 . 


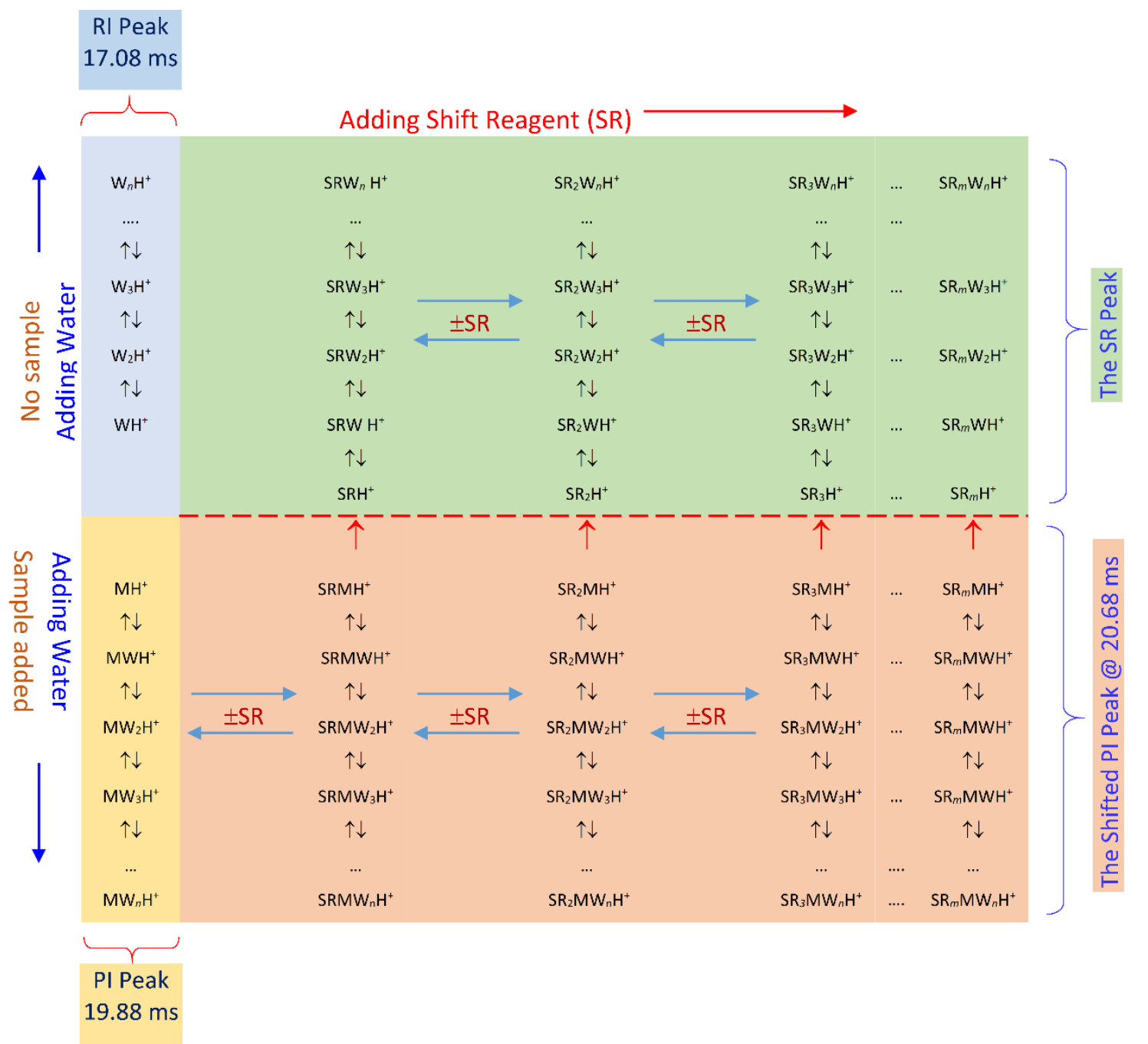

Fig.5 Possible ion molecule reactions and equilibria that may occur in the drift region, when sample (M) exists in the ionization chamber, and a shift reagent (SR) and water $(\mathrm{W})$ in both the ionization and the drift region. In the absence of sample and SR, only reactant ions of water clusters are formed in the ionization region, as shown in the top first column (blue section). When the sample is added to the ionization region, these ions convert into the product ions shown in the yellow section. This is the case for the peak at 19.88 in Fig. 4 corresponding to all hydrated protonated valinol $\left(\mathrm{VW}_{n} \mathrm{H}^{+}\right)$. If the shift reagent is added to the drift gas without sample, the green and blue sections show the nature of ions involved in making an ion mobility peak. If sample is added to the ionization region and at the same time SR is added to the buffer gas, the yellow and pink sections show ion-molecule reactions that ultimately generate the peak. This is the case for the peak at 20.68 that includes all protonated valinol clustered with water or 2-butanol $\left(\mathrm{B}_{m} \mathrm{VW}_{n} \mathrm{H}^{+}\right)$coalesced into a single mobility peak. Ammonium and higher order clusters of sample, $\mathrm{M}_{\mathrm{n}} \mathrm{H}^{+}$are not considered in these reactions.

It is assumed that sample is only added into the ionization region where the hydronium ions are produced, while water $(\mathrm{W})$ and the shift reagent $(\mathrm{SR})$ are present in both the reaction and drift regions. Ammonium ion and higher order clusters of sample, $\mathrm{M}_{\mathrm{n}} \mathrm{H}^{+}$are not considered in Fig. 5. The system is then described by considering several series of complex reactions or a multi-equilibria system that can proceed in two dimensions, adding a water or a shift reagent molecule to the core ion. This reaction map shows the species that may be observed at different conditions. In general, the peaks of four species may appear on the ion mobility spectrum. Water cannot be totally removed from the drift gas. Hence, in the absence of any sample and SR, (blue section) only water clusters of hydronium ion, $\mathrm{W}_{n} \mathrm{H}_{3} \mathrm{O}^{+}$, as the reactant ion peak, are observed. This is the case for Fig. 1 and 2 where the hydronium reactant ion peak is observed at $17.08 \mathrm{~ms}$. Above the dashed line no sample is present. If sample is added to the ionization region with no SR, (yellow section) only the first column below the dashed line describes the reactions happening in the drift region. Then, a mixture of water clusters of protonated sample $\left(\mathrm{MH}^{+} \mathrm{W}_{n}\right)$ makes the product ion peak (PIP). This is the case for Fig. 
4 a where a peak at $19.88 \mathrm{~ms}$ corresponding to water clusters of protonated valinol is observed. If the SR is added to drift region, without any sample, then the reactions proceed towards the right direction above the dashed line and occupy both the blue and the green section. In fact, a vector of $\mathrm{W}_{\mathrm{n}} \mathrm{H}^{+}$converts into a matrix. Then, the result is a mixture of $\mathrm{SR}_{m} \mathrm{~W}_{n} \mathrm{H}^{+}$ions with $n=1,2,3$.. and $m=0,1,2, \ldots$ Although this is not the case in our experiment, the peak at 223.4 amu in the mass spectrum presented in Fig. 3-b, corresponding to $\mathrm{B}_{3} \mathrm{H}^{+}$ is an example. When the sample is added to the ionization region and the SR is dopped into the drift gas, both water and SR play a role in the ion-molecule reactions and shift the distribution towards heavier $\mathrm{SR}_{m} \mathrm{MW}_{n} \mathrm{H}^{+}$ clusters. In fact, the protonated sample is siege by W and SR molecules. They quickly and frequently take off and on, so that the mobility gets smaller than the original protonated sample. In another word, the final mobility is a weighted average of all cluster ions. This cause the product ion peak shift towards longer drift times as demonstrated in Fig. 4, where the valinol peak shifted from 19.88 to $20.68 \mathrm{~ms}$. This peak corresponds to all ions bellow the dashed line (the yellow and peak sections). Nevertheless, if a valinol molecule is separated from the cluster ion, it jumps to the zone above the dashed line and the remaining ion is in the form of $\mathrm{SR}_{m} \mathrm{~W}_{n} \mathrm{H}$, such as $\mathrm{B}_{3} \mathrm{H}^{+}$in Fig. 3-b. This reaction. However, is one way since no valinol molecule is available in the drift region. Such ions may appear as a tail between the SR peak and the shifted PIP because they travelled partially as SR or PI. Obviously, the tail will not be observed if the two peaks are not separated well. Another possibility for observing such ion on the mass spectrometer could be the remaining unreacted $\mathrm{BH}^{+}$, formed in the ionization region, that uptake more $\mathrm{B}$ molecules on their way while traveling through a bath of B molecules. Examination of the ion mobility spectrum presented in Fig. 4-b show neither tail nor double peak. Hence, the SR peak appears the same drift time as the shifted PIP for valinol with 2-buthanol shift reagent.

In summary, all ions can be described either by one dimensional vectors, like the blue or yellow sections of the map, or two-dimensional matrices as described in the green and the pink sections. Adding the sample changes the vector $\mathrm{W}_{\mathrm{n}} \mathrm{H}^{+}$to a new vector of $\mathrm{MW}_{\mathrm{n}} \mathrm{H}^{+}$while adding the SR provides an extra dimension for expanding the vector to a matrix of $\mathrm{SR}_{m} \mathrm{MW}_{n} \mathrm{H}^{+}$. The same scenario can be imagined if ammonium ions are initially considered as the reactant ions. A separate matrix may also form from the dimer ion $\mathrm{M}_{2} \mathrm{H}^{+}$if the sample concentration is too high. Then a separated peak of the complex mixture of the dimer ion of $\mathrm{SR}_{m} \mathrm{M}_{2} \mathrm{~W}_{n} \mathrm{H}^{+}$, appears at higher drift time.

In the case of valinol as sample and 2-buthanol as shift reagent only few adducts, denoted by oval in Fig.5, including $\mathrm{BVH}^{+}$and $\mathrm{B}_{2} \mathrm{VH}^{+}$where observed. Other adducts with a larger number of 2-butanol molecules are less abundant and cannot be seen in the mass spectra but they have been reported (Bollan et al. 2007; Fernandez-Maestre et al. 2010b). The electrostatic surface potential map for one of the SR used in this study, trifluoromethyl benzyl alcohol (F), with methionine, F-MetH ${ }^{+}$, is shown in Figure S3 (Supplementary Information). This map demonstrates that the nucleophilic regions on the SR disappear after clustering with methionine. The electrophilic regions remain on the cluster for additional adduction.

\subsection{Mobility shifts after the formation of SR-ion adducts}

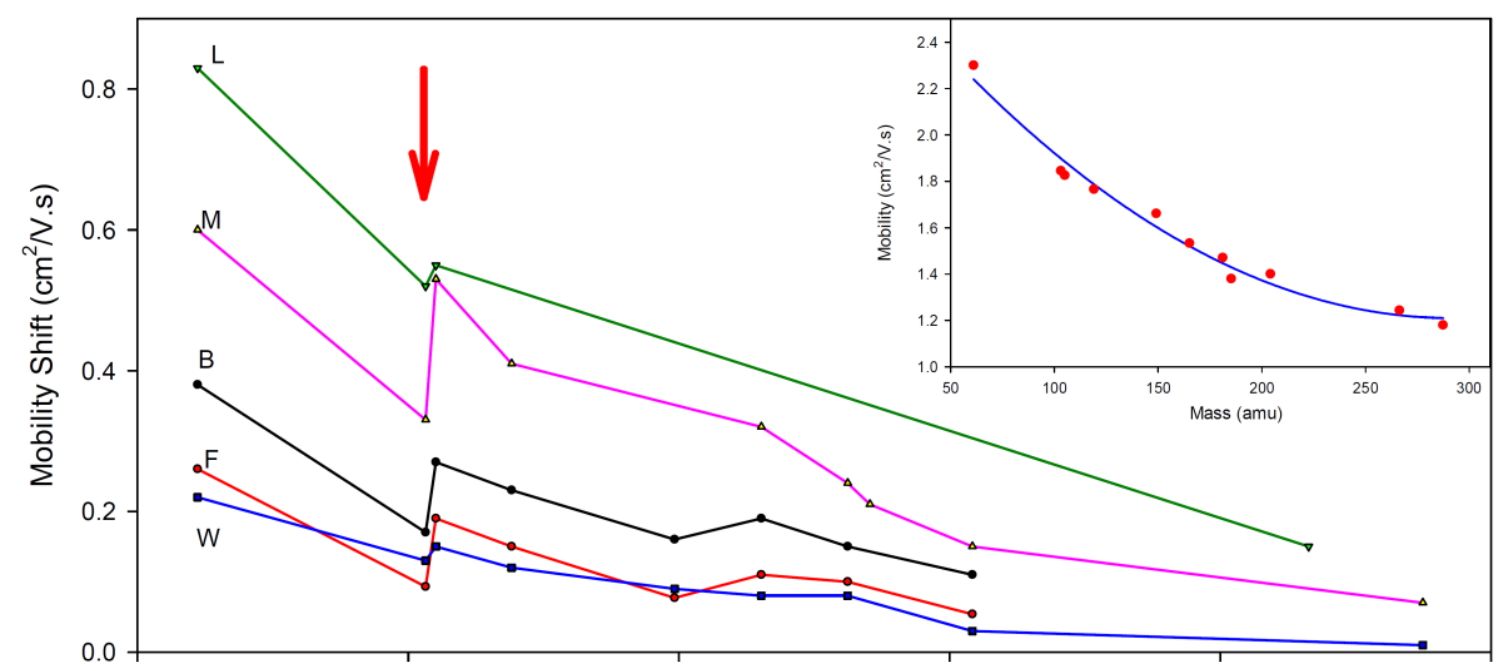




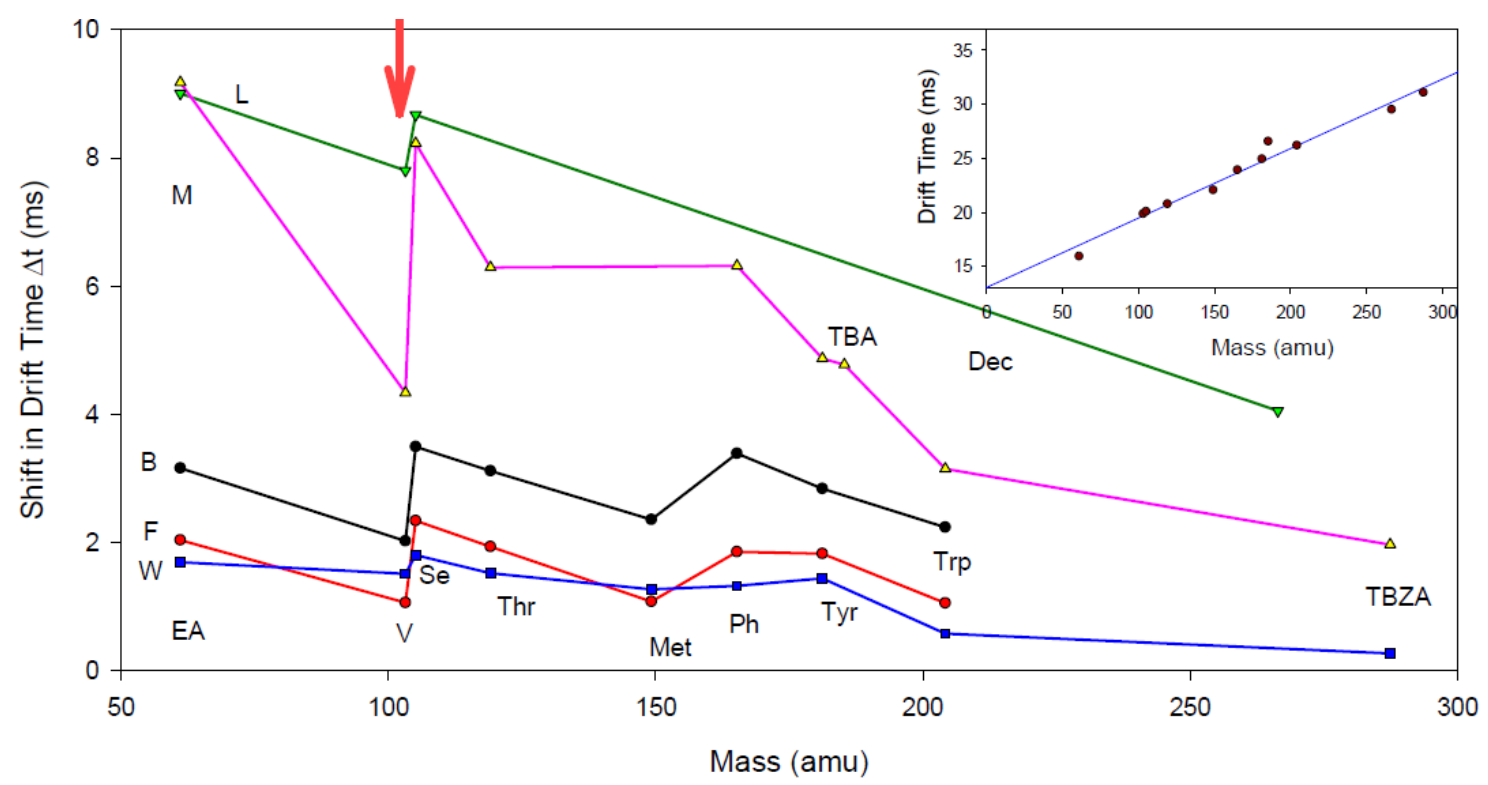

Fig. 6 a) Mobility and b) drift time shifts of ethanolamine (Et), valinol (V), serine (S), threonine (Thr), phenylalanine $(\mathrm{P})$, tyrosine (Tyr), tributylamine (Tb), tryptophan (Try), desipramine (D), and tribenzylamine (Tz), when different SR were introduced into the buffer gas at $150^{\circ} \mathrm{C}$. The concentrations of SR in the buffer gas were $2.3,1.0,879,1.7$, and $6.8 \mathrm{mmol} \mathrm{m-3}$, for $\mathrm{F}, \mathrm{M}, \mathrm{W}, \mathrm{L}$, and $\mathrm{B}$, respectively. $\mathrm{R}^{2}$ regression coefficients show the mobility shift-mass correlations. Table 1 shows the numeric values of these mobility shifts and SR concentration. B: 2-butanol, F: trifluoromethyl benzyl alcohol, L: ethyl lactate, M: methyl-2-chloro propionate, $\mathrm{W}$ : water. The analytes in the graphs, from bottom to top, are: $\mathrm{M}=\mathrm{Et}, \mathrm{V}, \mathrm{S}, \mathrm{Thr}, \mathrm{P}, \mathrm{Tyr}, \mathrm{Tb}$, Try, Tz; F = Et, V, S, Thr, P, Tyr, Try; B = Et, V, S, Thr, P, Tyr, Try; W = Et, V, S, Thr, Met, P, Tyr, Try, Tz; L = Et, $\mathrm{V}, \mathrm{S}, \mathrm{D}$.

Figure 6 shows the shifts in mobility and drift time of selected ions, ethanolamine, valinol, serine, threonine, phenylalanine, tyrosine, tributylamine, tryptophan, desipramine, and tribenzylamine, when different SR were introduced into the buffer gas at different concentrations. Table 1 shows these mobility shift values when the SR concentration was increased from $0.0 \mathrm{mmol} \mathrm{m}^{-3}$ to the specific concentration shown in this table at 150 ${ }^{\circ} \mathrm{C}$. The onsets show the behavior of the analyte versus mass mobility and drift time. It can be seen that the drift time linearly depends on the mass, while the mobility does not. This is due to the fact that drift time depends on the size of the ion. For a homologous series, the size is determined by the number of atoms and it is directly related to mass. The mobility itself is inversely proportional to drift time. Therefore, mobility is not expected to be a linear function of mass. The inverse relation between the mobility and drift time also affects the graphs of the shift versus mass. The plots in Fig 6 show that, both shifts, in mobility and in drift time, have similar decreasing trends with mass, but higher slopes for $\Delta K$ are observed. The steeper plots for $\Delta K$ may be explained by considering Eq. 2 .

$$
K \propto \frac{1}{t} \Rightarrow \Delta K \propto \frac{\Delta t}{t^{2}}
$$

Based on the plot shown in the onset of Fig. 6-b, the drift time $t$ may be substituted with mass $m$. Hence, we have,

$$
\Delta K \propto \frac{\Delta t}{(m+b)^{2}}
$$

Where $b$ is the intercept of the drift time-mass plot. Eq. 3 shows the relationship between the mobility shift and the drift time shift. Assuming a decreasing $\Delta t$ with mass, it is evident that $\Delta K$ decreases more rapidly than $\Delta t$, since the squared $m$ exists in the denominator of the right hand side of Eq. 3. This means that, even if the effect of a specific SR on drift times of ions with different masses are similar, or in another word, if the peak 
displacement, $\Delta t$, is constant, $\Delta K$ still decreases with mass. This may mask the effect of SR on a series of ions with different masses. Hence, unlike traditional way, we focus on $\Delta t$ rather than $\Delta K$. In fact, $\Delta t$ purely reflects the changes in size of an ion due to the presence of SR.

All the plots in Fig. 6-a show a general negative slope. This means that the largest drift time shifts were obtained by the compounds of lower mass or smaller size, in this case, ethanolamine (average $\Delta t 5.01 \mathrm{~ms}$ ) and serine $(\Delta t 4.9 \mathrm{~ms})$ and the smallest shift by the largest mass compound, tribenzylamine $(\Delta t 1.11 \mathrm{~ms})$. This is because the larger the mass or size of an ion, the less its size is affected by the adduction of molecules. Average $\Delta t$ showed correlation with the ion masses with a $0.70 \mathrm{R}^{2}$ regression coefficient.

In a homologous series, the mass determines the position of an ion in the mobility or time shift-mass correlation plots when a SR has been injected into the buffer gas in IMS (Figure 5). However, this correlation is affected by the presence of intramolecular bonds and by the interaction energy with the SRs (FernandezMaestre 2018). It is clear in Fig. 6 that, methionine departed from these correlations because it is less influenced by the introduction of a SR due to the formation of an intramolecular bond that hinders the adduction with the SR, because the positive charge of the ions is delocalized, and also due to the steric hindrance on the ion's charge (Nieckarz et al. 2008; Fernandez-Maestre et al. 2012). Karpas (1989) showed that protonated $\alpha, \omega$-diamines have their charge delocalized due to an intramolecular hydrogen bond. This led to more condensed structures than those of the protonated primary n-amines increasing the diamines' mobility more than that of n-amines. Also, due to the formation of intramolecular hydrogen bonds, it was found that $\alpha, \omega$-diamines had a lesser interaction with 18C6 than n-amines (Parchami et al. 2017b). In the graph for water, it is hard to demonstrate a departure from linearity for methionine because its differences with the ion mobilities of tyrosine and phenylalanine were not statistically significant maybe because the experiments were performed at very high flow rates, $1250 \mu \mathrm{L} / \mathrm{hr}$. At these high flow rates, the content of the $0.25 \mathrm{ml}$ syringe was emptied in only 12 minutes, imposing limited reproducibility to the experimental conditions because the time to reach a homogeneous saturation of water in the drift gas was short.

Table 1. Mobility shifts, $\Delta \mathrm{K}_{0}$, and drift time shifts $\Delta t$, for the selected ions when different SR were introduced into the buffer gas at 2.3, 1.0, 879, 1.7, and $6.8 \mathrm{mmol} \mathrm{m}^{-3}$ concentrations for F, M, W, L, and B, respectively. $\Delta \mathrm{K}_{0}$ values were calculated as the mobility difference in nitrogen-only buffer gas and SR-doped buffer gas. Average $\Delta \mathrm{K}_{0}$ showed correlation with the ion masses with a $0.73 \mathrm{R}^{2}$ regression coefficient (FernandezMaestre et al. 2010b, 2012).

\begin{tabular}{|c|c|c|c|c|c|c|c|c|c|c|c|c|}
\hline \multirow{2}{*}{ Ion } & \multicolumn{10}{|c|}{ Shift reagent } & \multirow{4}{*}{\multicolumn{2}{|c|}{ 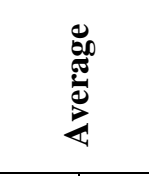 }} \\
\hline & \multicolumn{2}{|c|}{$\mathbf{B}^{\text {a }}$} & \multicolumn{2}{|c|}{$\mathbf{F}^{\mathbf{b}}$} & \multicolumn{2}{|c|}{$\mathbf{L}^{\mathbf{c}}$} & \multicolumn{2}{|c|}{$\mathbf{M}^{\mathbf{b}}$} & \multicolumn{2}{|c|}{$\mathbf{W}^{\mathbf{b}}$} & & \\
\hline SR mass & \multicolumn{2}{|c|}{74.1} & \multicolumn{2}{|c|}{176.1} & \multicolumn{2}{|c|}{118.1} & \multicolumn{2}{|c|}{122.6} & \multicolumn{2}{|c|}{18.0} & & \\
\hline $\mathrm{SR}, \mathrm{mmol} \mathrm{m}^{-3}$ & \multicolumn{2}{|c|}{6.8} & \multicolumn{2}{|c|}{2.3} & \multicolumn{2}{|c|}{1.7} & \multicolumn{2}{|c|}{1.0} & \multicolumn{2}{|c|}{879} & & \\
\hline Shift & $-\Delta K$ & $\Delta t$ & $-\Delta K$ & $\Delta t$ & $-\Delta K$ & $\Delta t$ & $-\Delta K$ & $\Delta t$ & $-\Delta K$ & $\Delta t$ & $-\Delta K$ & $\Delta t$ \\
\hline Ethanolamine & 0.38 & 3.16 & 0.26 & 2.03 & 0.83 & 9.00 & 1.37 & 9.17 & 0.22 & 1.69 & 0.67 & 5.01 \\
\hline Valinol & 0.17 & 2.02 & 0.09 & 1.05 & 0.52 & 7.80 & 0.33 & 4.33 & 0.13 & 1.51 & 0.27 & 3.34 \\
\hline Serine & 0.27 & 3.49 & 0.19 & 2.33 & 0.55 & 8.67 & 0.53 & 8.22 & 0.15 & 1.80 & 0.36 & 4.90 \\
\hline Threonine & 0.23 & 3.11 & 0.15 & 1.93 & - & -- & 0.41 & 6.29 & 0.12 & 1.51 & 0.23 & 3.21 \\
\hline Methionine & 0.16 & 2.35 & 0.077 & 1.07 & - & -- & - & -- & 0.09 & 1.26 & 0.08 & 1.56 \\
\hline Phenylalanine & 0.19 & 3.38 & 0.11 & 1.85 & - & -- & 0.32 & 6.31 & 0.08 & 1.32 & 0.17 & 3.22 \\
\hline Tyrosine & 0.15 & 2.83 & 0.10 & 1.82 & - & -- & 0.24 & 4.87 & 0.08 & 1.434 & 0.14 & 2.74 \\
\hline Tributylamine & - & -- & - & -- & - & -- & 0.21 & 4.77 & - & -- & - & - \\
\hline Tryptophan & 0.11 & 2.23 & 0.05 & 1.051 & - & -- & 0.15 & 3.14 & 0.03 & 0.58 & 0.08 & 1.75 \\
\hline Desipramine & - & -- & - & -- & 0.15 & 4.05 & - & -- & - & -- & - & - \\
\hline Tribenzylamine & - & -- & - & -- & - & -- & 0.07 & 1.96 & -0.01 & 0.26 & 0.04 & 1.11 \\
\hline Average & & 2.82 & & 1.64 & & 7.38 & & 5.45 & & 1.26 & & \\
\hline
\end{tabular}

B: 2-butanol, F: trifluoromethyl benzyl alcohol, L: ethyl lactate, M: methyl-2-chloro propionate, W: water. ${ }^{\text {a }}$ Fernandez-Maestre et al. 2010b. ${ }^{b}$ Fernandez-Maestre et al. 2010a. ${ }^{\mathrm{c}}$ Fernandez-Maestre et al. 2012. More SR and ions are shown in Table S1. 


\subsection{The interaction energy of SR-ion adducts}

The departure from linearity of the other compound, valinol, is more evident in Figure 6 (solid arrows) where the shift-mass lines suffer a strong turn from valinol to serine to retake linearity after this amino acid. Valinol is a small molecule with little steric hindrance and the explanation for its departure from linearity is the interaction energy (Table 2). The lifetimes of the clusters depend on the interaction energies: the higher the interaction energy the larger the lifetime. A larger adduct lifetime increases the average size of the ion because it travels the drift tube a long time as a large adduct. A larger size increases the ions' collisions with the buffer gas, decreasing the ion mobilities. The strongest interaction energy of the ion:SR adducts originates from the hydrogen bonds formed between the positive charge of the ion and electron-rich groups on the SR. For valinol, the ion interaction energies with any of the SR used were lower than those for serine. This implied that valinol had a shorter adduct lifetime, a smaller average size, and fewer collisions with the buffer gas. Therefore, the mobility of valinol was less affected than those of the other ions and its mobility shifts were also smaller than those of the other ions (Figure 5).

The origin of the higher interaction energy of serine with any SR, when compared to valinol, resides in the fact that serine is an amino acid and has one additional $\mathrm{OH}$ and carboxylic groups. These groups yield a stronger interaction energy of serine with most SR (Table 2). The effects of intramolecular hydrogen bonding, interaction energy, and proton affinity on ion mobility after the injection of SR have been reviewed (Fernandez-Maestre 2018; Waraksa et al. 2016).

Table 2 shows the interaction energies for the compounds and adducts investigated, and Table S3 their interaction energies, Gibbs energies, enthalpies and entropies. The Gibbs energy describes the condition of equilibrium and spontaneity in a process. All Gibbs energies were negative indicating that the complexation processes were exergonic and spontaneous for the formation of clusters in all species corroborating the interaction energies calculations.

Table 2. Interaction energies (IE) in $\mathrm{kcal} / \mathrm{mol}$ for the ions and adducts investigated. Calculations were made using Gaussian 09 (Revision D.01) at $150^{\circ} \mathrm{C}$ and the X3LYP-GD3/6-311++(d,p) functional. The complete data set with energies in Hartree and $\mathrm{kJ} / \mathrm{mol}$ is found in Table S2.

\begin{tabular}{|l|c|c|c|c|c|c|}
\hline \multirow{2}{*}{ Analytes } & \multicolumn{5}{|c|}{ Shift Reagents } & \\
\cline { 2 - 7 } & $\mathbf{B}^{\mathbf{a}}$ & $\mathbf{F}^{\mathbf{b}}$ & $\mathbf{L}^{\mathbf{c}}$ & $\mathbf{M}^{\mathbf{b}}$ & $\mathbf{W}^{\mathbf{b}}$ & Average \\
\hline Ethanolamine & -20.2 & -19.8 & -28.8 & -25.1 & -17.2 & -22.2 \\
\hline Valinol & -17.1 & -17.1 & -25.2 & -21.7 & -14.6 & -19.1 \\
\hline 2-methyl-3-pentanamine & -17.9 & -17.8 & -26.1 & -22.4 & -15.2 & -24.8 \\
\hline Serine & -23.9 & -21.1 & -40.8 & -35.4 & & -30.3 \\
\hline Threonine & -19.6 & -17.6 & & -24.2 & -16.5 & -19.5 \\
\hline Phenylalanine & -20.7 & -19.4 & & -33.5 & -16.7 & -22.6 \\
\hline Tyrosine & -20.0 & -19.2 & & -24.8 & -17.2 & -20.3 \\
\hline Tributylamine & -19.6 & & & & & -19.6 \\
\hline Tryptophan & -18.9 & -18.8 & & -22.7 & -16.3 & -19.2 \\
\hline Desipramine & & & -27.8 & & & -27.8 \\
\hline Average & -19.8 & -18.8 & -29.7 & -26.2 & -16.2 & \\
\hline
\end{tabular}

B: 2-butanol, F: trifluoromethyl benzyl alcohol, L: ethyl lactate, M: methyl-2-chloro propionate, W: water.

A compound, 2-methyl-3-pentanamine, was compared to valinol, of similar structure, concerning the interaction energy with the SR (Table 2). The difference of 2-methyl-3-pentanamine with valinol is that the $\mathrm{OH}$ group in valinol is replaced by a methyl group (Figure S2). It would be expected that the presence of an $\mathrm{OH}$ group in valinol would produce a stronger bond with all SRs than 2-methyl-3-pentanamine based on the comparison made above between valinol and serine. However, in all cases, the opposite result was obtained (Table 2). The reason for this result is the presence of an intramolecular bond in valinol with a $-9.2 \mathrm{kcal} / \mathrm{mol}$ energy between the positive charge on the nitrogen and the free electrons of the oxygen (Figure S5). This bond hinders the interaction of serine with 2-butanol, as has been demonstrated with atenolol, (Fernandez-Maestre 2018) by dispersing the positive charge on the nitrogen over a larger number of atoms. This bond is not present in 2-methyl-3-pentanamine because of the absence of the hydroxyl group. Figure S6 shows the potential energy surface maps when valinol protonation is carried out. Upon protonation of valinol, the intramolecular 
bond is formed. If we force the anti-position between the amino group and the hydroxyl group when protonation is carried out, we can observe a $\sim 12 \mathrm{kcal} / \mathrm{mol}$ difference in the stabilization energy of the molecule, indicating that the structure with the higher stabilization energy, forming the intramolecular bond, is the most favored. When valinol was excluded from the graphs in Figure 6-b, the $\mathrm{R}^{2}$ correlation coefficients increased from $0.58,0.70,0.76,0.90$, and 0.91 to $0.68,0.89,0.93,0.90$, and 0.93 , for $\mathrm{M}, \mathrm{F}, \mathrm{B}, \mathrm{W}$, and $\mathrm{L}$ shift reagents, respectively, indicating the extent of the departure of valinol from linearity and the effect of the low cluster interaction energy.

\subsection{Average shifts and interaction energies}

Fig. 6 shows that the shift is a function of mass or size with negative slope. However, the slope differs for various SRs. Water gives the smallest shift while ethyl lactate shows the largest shift. The smallest shifts are produced by $\mathrm{W}, \mathrm{B}$ and $\mathrm{F}$ for all analytes and the largest ones are produced by $\mathrm{L}$ and $\mathrm{M}$. This is perhaps due to the presence of $\mathrm{C}=\mathrm{O}$ bond in $\mathrm{L}$ and $\mathrm{M}$ that generates higher proton affinity than other SRs. Also, these SRs have three interaction sites compared to one site on the other SR. The effect of SR on the shifts can be more accurately discovered if the interaction energy between the SR and the ion is considered. Fig. S7 shows a large correlation $\left(\mathrm{R}^{2}>0.98\right)$ between the average interaction energies and the average shifts for the selected SRs and analytes. In fact, the shift depends not only to the individual analyte, but also to the tendency of the SR for attachment.

\section{CONCLUSIONS}

We observed time and mobility shift-mass correlations for protonated desipramine, ethanolamine, serine, methionine, phenylalanine, threonine, tribenzylamine, tributylamine, tryptophan, tyrosine, and valinol when different SR were introduced into the buffer gas at $150{ }^{\circ} \mathrm{C}$. This correlation is due to the fact that the lighter or smaller ions are more affected by adduction of the SR than the heavier or larger ions. However, the correlation had exceptions in our experiments: valinol, due to the low binding energy with the SR, and methionine, due to the formation of an intramolecular hydrogen bond. Furthermore, it was shown that the average shifts perfectly correlate to the average interaction energies. This shows that the shift is determined by the initial size of the ion as well as the SR-ion interaction. The understanding of this behavior is important in IMS to predict the adequate SR for a given interferent in the detection of illegal substances in airports, ports, and customs. These interferents cause false positives that hinder the transit of passengers and cargo due to unnecessary deeper inspections. SRs can be used to rule out the presence of false positives by facilitating transport and trade.

\section{ACKNOWLEDGEMENTS}

The corresponding author thanks the kind help of Herbert Hill and Bill Siems in WSU for their tutoring when obtaining this data, this article is dedicated to them,. Financial support was provided by a Fulbright scholarship, Universidad de Cartagena, Washington State University, and Herbert Hill lab. Experiments were performed at Washington State University.

\section{Declarations}

Funding Not applicable

Conflicts of interest/Competing interests. The authors declare that they have no known competing financial interests or personal relationships that could have appeared to influence the work reported in this paper.

Availability of data and material. The full dataset is available in reference (Fernandez-Maestre 2017) Authors' contributions Meza did the theoretical studies and Fernandez the experiments. The first draft of the manuscript was written by Fernandez and all authors commented on previous versions of the manuscript. All authors read and approved the final manuscript.

\section{REFERENCES}

Bahrami H, Tabrizchi M (2012) Combined corona discharge and UV photoionization source for ion mobility spectrometry. Talanta 97:400-405. https://doi.org/10.1016/j.talanta.2012.04.052

Bollan HR, Stone JA, Brokenshire JL, Rodriguez JE, Eiceman GA (2007) Mobility Resolution and Mass Analysis of Ions from Ammonia and Hydrazine Complexes with Ketones Formed in Air at Ambient Pressure. J Am Soc Mass Spectrom 18:940-951. https://doi.org/10.1016/j.jasms.2007.01.014 
Butcher D, Miksovska J, Ridgeway ME, Park MA, Fernandez-Lima F (2019) The effects of solution additives and gas-phase modifiers on the molecular environment and conformational space of common heme proteins. Rapid Comm Mass Spectrom 33:399-404. https://doi.org/10.1002/rcm.8347

Campbell JL, Zhu M, Hopkins WS (2014) Ion-Molecule Clustering in Differential Mobility Spectrometry: Lessons Learned from Tetraalkylammonium Cations and their Isomers. J Am Soc Mass Spectrom 25:15831591. https://doi.org/10.1007/s13361-014-0939-3

Cohen MJ, Karasek FW (1970) J Chromatogr Sci 8:330-337. https://doi.org/10.1093/chromsci/8.6.330

Fernandez-Maestre, R., Harden, C. S., Ewing, R. G., Crawford C. L., Hill Jr HH (2010a) Chemical Standards in Ion Mobility Spectrometry. Analyst [London] 135:1433-1442. https://doi.org/10.1039/B915202D

Fernandez-Maestre R, Wu C, Hill Jr HH (2010b) Using a buffer gas modifier to change separation selectivity in ion mobility spectrometry. Int J Mass Spectrom 298:2-9. https://doi.org/10.1016/j.ijms.2010.08.009

Fernandez-Maestre R, Wu C, Hill Jr HH (2012) Buffer gas modifiers effect resolution in ion mobility spectrometry through selective ion-molecule clustering reactions. Rapid Commun Mass Spectrom 26:1-13. https://doi.org/10.1002/rcm.6335

Fernandez-Maestre R (2017) Accuracy of reduced mobilities and measurement of instrumental parameters in ion mobility spectrometry. Int J Mass Spectrom 421:8-13. https://doi.org/10.1016/j.ijms.2017.05.018

Fernandez-Maestre R (2018) Buffer gas additives (modifiers/shift reagents) in ion mobility spectrometry: Applications, predictions of mobility shifts, and influence of interaction energy and structure. J Mass Spectrom 53:598-613. https://doi.org/10.1002/jms.4190

Eiceman GA, Salazar MR, Rodriguez MR, Limero TF, Beck SW, Cross JH, Young R, James JT (1993) Ion Mobility Spectrometry of Hydrazine, Monomethylhydrazine, and Ammonia in Air with 5-Nonanone Reagent Gas. Anal Chem 65:1696-1702. https://doi.org/10.1021/ac00061a011

Eiceman GA, Kelly K, Nazarov EG (2002) Nitric oxide as a reagent gas in ion mobility spectrometry. Int J Ion Mobil Spectrom 5:22-30

Eiceman GA, Stone JA (2004) Ion mobility spectrometers in national defense. Anal Chem 76:390-397A. https://doi.org/10.1021/ac041665c

Foresman JB, Frisch A (1996) Exploring Chemistry with Electronic Structure Methods. Gaussian, Inc. Pittsburgh, PA, USA, ISBN-13: 978-0963676931

Frisch MJ, Trucks GW, Schlegel HB, Scuseria GE, Robb MA, Cheeseman JR, et al. (2009) Gaussian 09 Revision B.01 Gaussian, Inc. Wallingford, CT.

Ghaemi E, Alizadeh N (2014) Nanoclusters formation in ion mobility spectrometry and change separation selectivity of picoline isomers. Int J Ion Mobil Spectrom 17:117-124. https://doi.org/10.1007/s12127-014$\underline{0157-5}$

Jeanne Dit Fouque K, Moreno J, Fernandez-Lima F (2019) Exploring the Conformational Space of GrowthHormone-Releasing Hormone Analogues Using Dopant Assisted Trapped Ion Mobility Spectrometry-Mass Spectrometry. J Phys Chem B 123:6169-6177. https://doi.org/10.1021/acs.jpcb.9b03777

Karpas Z (1989) Evidence for proton-induced cyclization in $\alpha$-w diamines from mobility measurements. Int J Mass Spectrom Ion Proc 93:237-242. https://doi.org/10.1016/0168-1176(89)80100-4

Levin DS, Vouros P, Miller RA, Nazarov EG (2007) Using a nanoelectrospray-differential mobility spectrometer-mass spectrometer system for the analysis of oligosaccharides with solvent selected control over ESI aggregate ion formation. J Am Soc Mass Spectrom 18:502-511.

https://doi.org/10.1016/j.jasms.2006.10.008 
Nieckarz RJ, Atkins CG, McMahon TB (2008) Effects of Isomerization on the Measured Thermochemical Properties of Deprotonated Glycine/Protic-Solvent Clusters. Chem Phys Chem 9:2816- 2825.

https://doi.org/10.1002/cphc.200800525

Oberreit D, Rawat VK, Larriba-Andaluz C, Ouyang H, McMurry PH, Hogan Jr CJ (2015) Analysis of heterogeneous water vapor uptake by metal iodide cluster ions via differential mobility analysis-mass spectrometry. J Chem Phys 143:104204. https://doi.org/10.1063/1.4930278

Parchami R, Kamalabadi M, Alizadeh N (2017a) Determination of biogenic amines in canned fish samples using head-space solid phase microextraction based on nanostructured polypyrrole fiber coupled to modified ionization region ion mobility spectrometry. J Chrom A 1481:37-43. https://doi.org/10.1016/j.chroma.2016.12.046

Parchami R, Tabrizchi M, Alizadeh N (2017b) Effect of intramolecular hydrogen bonding of $\alpha, \omega$-diamines on the structure and exchange affinity of 18-crown-6-amine host-guest complexes in gas phase: A collision cross section measurements by ion mobility spectrometry. Int J Mass Spectrom Volume 421, Pages 150-155. https://doi.org/10.1016/j.ijms.2017.06.013

Puton J, Nousiainen M, Sillanpaa M (2008) Ion mobility spectrometers with doped gases. Talanta 76:978987. https://doi.org/10.1016/j.talanta.2008.05.031

Rawat VK, Vidal-de-Miguel G, Hogan CJ (2015) Modeling vapor uptake induced mobility shifts in peptide ions observed with transversal modulation ion mobility spectrometry-mass spectrometry. Analyst 140:69456954. https://doi.org/10.1039/C5AN00753D

Roscioli KM, Lamabadusuriya MR, Harden CS, Midey AJ, Wu C, Siems WF, Hill Jr HH (2014) Structure selective ion molecule interactions (SSIMI) in ion mobility spectrometry. Int J Ion Mobil Spectrom 17:4353. https://doi.org/10.1007/s12127-013-0143-3

Tsai CW, Midey A, Wu C, Yost RA (2016) Analysis of ammonium nitrate/urea nitrate with crown ethers and sugars as modifiers by electrospray ionization-mass spectrometry and ion mobility spectrometry. Analytical chemistry, 88:9435-9442. https://doi.org/10.1021/acs.analchem.6b01322

Vautz W, Nolte J, Fobbe R, Baumbach JI (2009) Breath analysis—performance and potential of ion mobility spectrometry. J Breath Res 3:036004. https://doi.org/10.1088/1752-7155/3/3/036004

Waraksa E, Perycz U, Namieśnik J, Sillanpää M, Dymerski T, Wójtowicz M, Puton J (2016) Dopants and gas modifiers in ion mobility spectrometry. TrAC Trends Anal Chem 82:237-249.

https://doi.org/10.1016/j.trac.2016.06.009

Wittmer D, Chen YH, Luckenbill BK, Hill HH (1994) Electrospray ionization ion mobility spectrometry. Anal Chem 66:2348-2355. https://doi.org/10.1021/ac00086a021 


\section{Supplementary Information International Journal of Mass Spectrometry}

\section{Ion-shift reagent binding energy and the shift-mass correlation in ion mobility spectrometry}

Roberto Fernandez-Maestre, ${ }^{\mathrm{a}, \mathrm{b}}$ Mahmoud Tabrizchi, ${ }^{\mathrm{c}}$ and Dairo Meza-Morelos ${ }^{\mathrm{a}^{*}}$

${ }^{a}$ Universidad de Cartagena, Campus de San Pablo, Programa de Química, Cartagena, Colombia

*dmezam@unicartagena.edu.co

${ }^{\mathrm{b}}$ National Research Nuclear University MEPhI (Moscow Engineering Physics Institute). Kashirskoe sh. 31, Moscow, 115409, Russia

${ }^{c}$ Department of Chemistry, Isfahan University of Technology, Isfahan 84156-83111, Iran
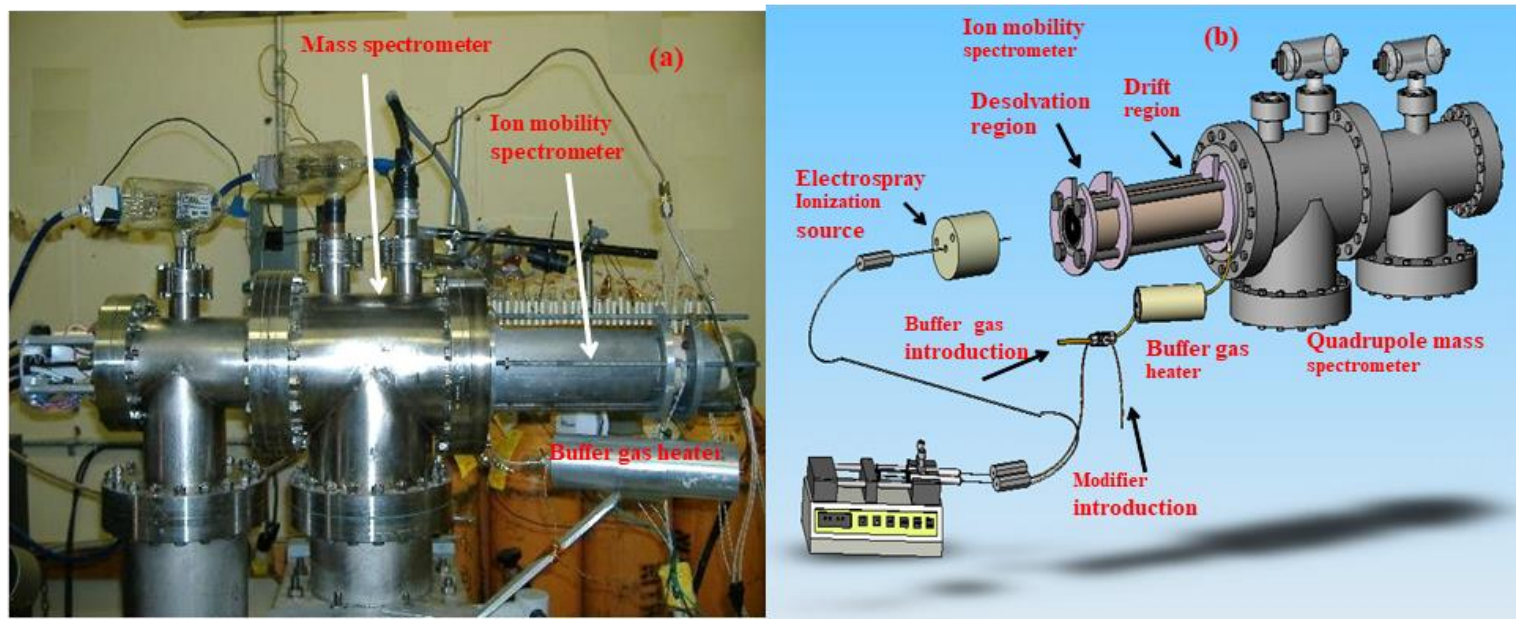

Fig. S1 Photograph of the IMS-MS instrument. 

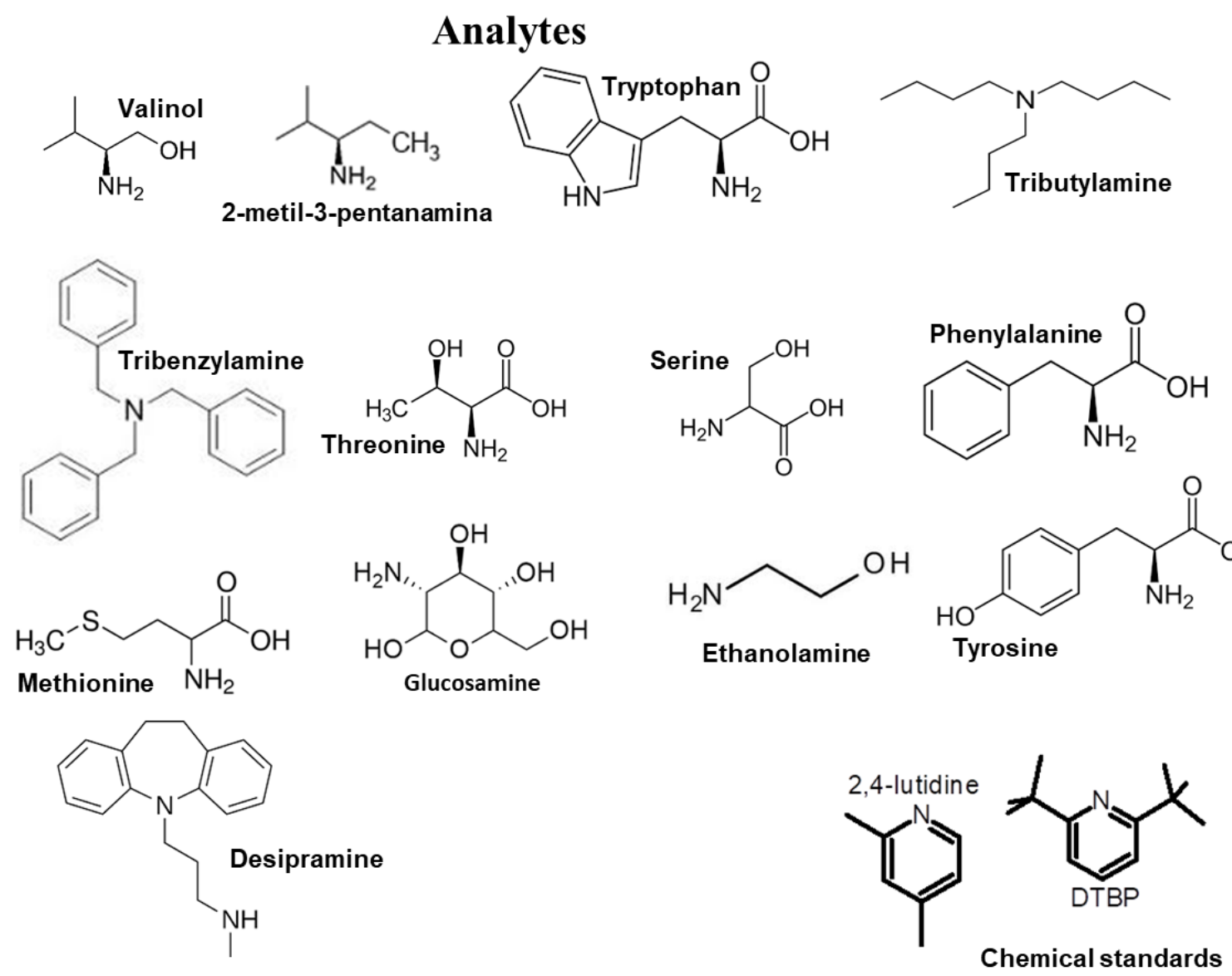

\section{Shift reagents}<smiles>CCOC(=O)C(C)O</smiles>

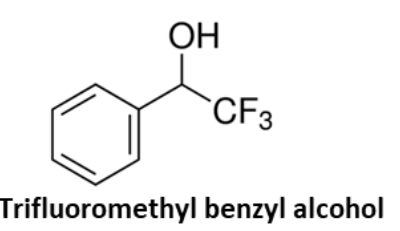<smiles>[Z16]CC(C)O</smiles>

Fig. S2 Structures of compounds used in this investigation. 


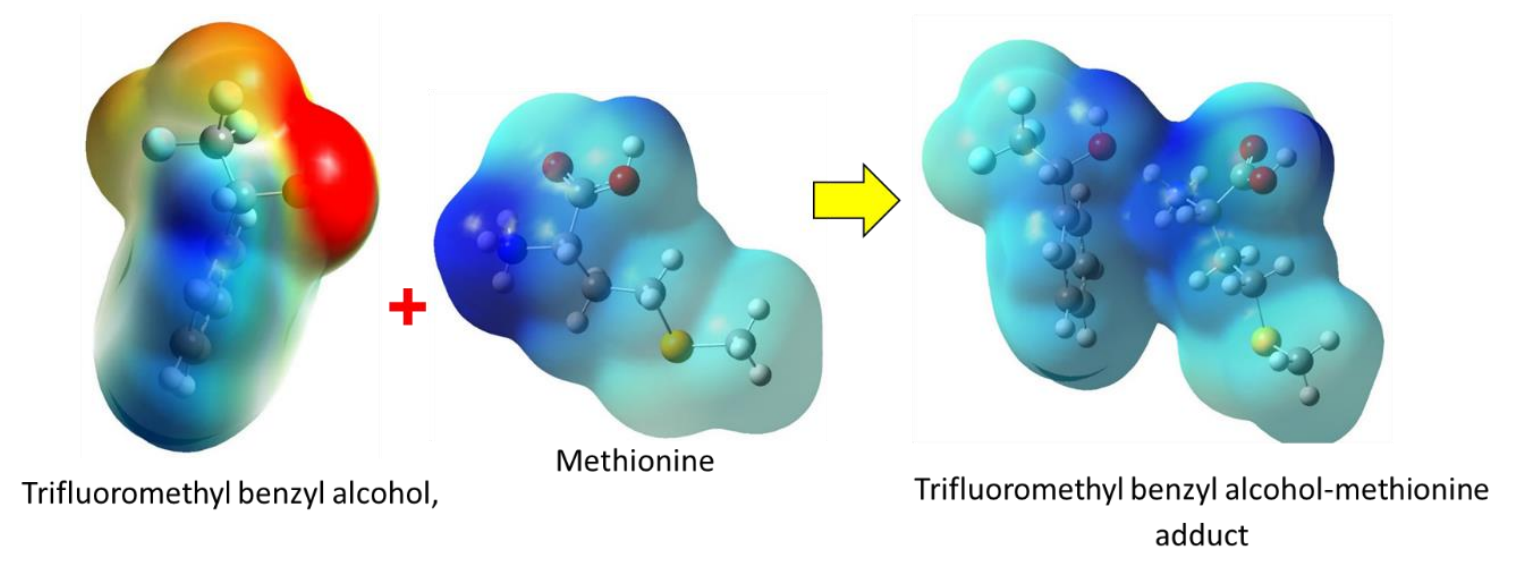

Fig. S3 Electrostatic surface potential map (ESPM) for the adduct trifluoromethyl benzyl alcohol-methionine demonstrating that the red nucleophilic regions in the SR ESPM disappeared after adduction and electrophilic regions remained in the adduct for additional adduction. 

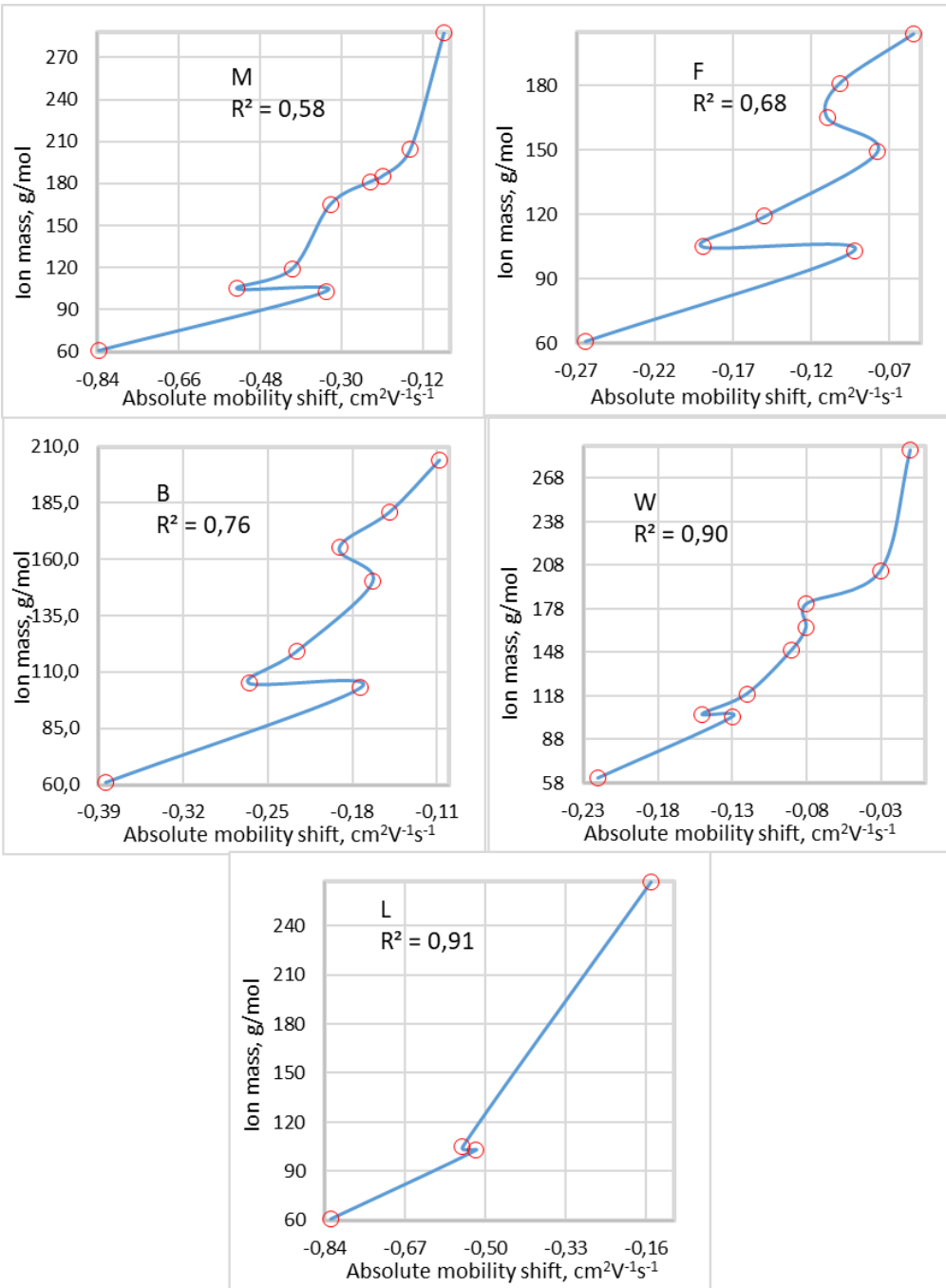

Fig. S4 Mobility shifts of ethanolamine, valinol (blue solid arrow), serine, threonine, methionine (red dotted arrow), phenylalanine, tyrosine, tributylamine, tryptophan, desipramine, and tribenzylamine, when different SR were introduced into the buffer gas at $150{ }^{\circ} \mathrm{C}$. Table 1 shows the numeric values of these mobility shifts. The concentrations of SR in the buffer gas were 2.3, 1.0, 879, 1.7, and $6.8 \mathrm{mmol} \mathrm{m}-3$, for F, M, W, L, and B, respectively. The analytes in the graphs, from bottom to top, are: $\mathrm{M}=\mathrm{Et}, \mathrm{V}, \mathrm{S}, \mathrm{Thr}, \mathrm{P}, \mathrm{Tyr}, \mathrm{Tb}, \mathrm{Try}, \mathrm{Tz} ; \mathrm{F}=$ Et, V, S, Thr, Met, P, Tyr, Try; B = Et, V, S, Thr, Met, P, Tyr, Try; W = Et, V, S, Thr, Met, P, Tyr, Try, Tz; L = Et, V, S, D. B: 2-butanol, D: desipramine, Et: ethanolamine, F: trifluoromethyl benzyl alcohol, L: ethyl lactate, M: methyl-2-chloro propionate, Met: methionine, P: phenylalanine, S: serine, Tb: tributylamine, Thr: threonine, Try: tryptophan, Tyr: tyrosine, Tz: tribenzylamine, V: valinol, W: water. 


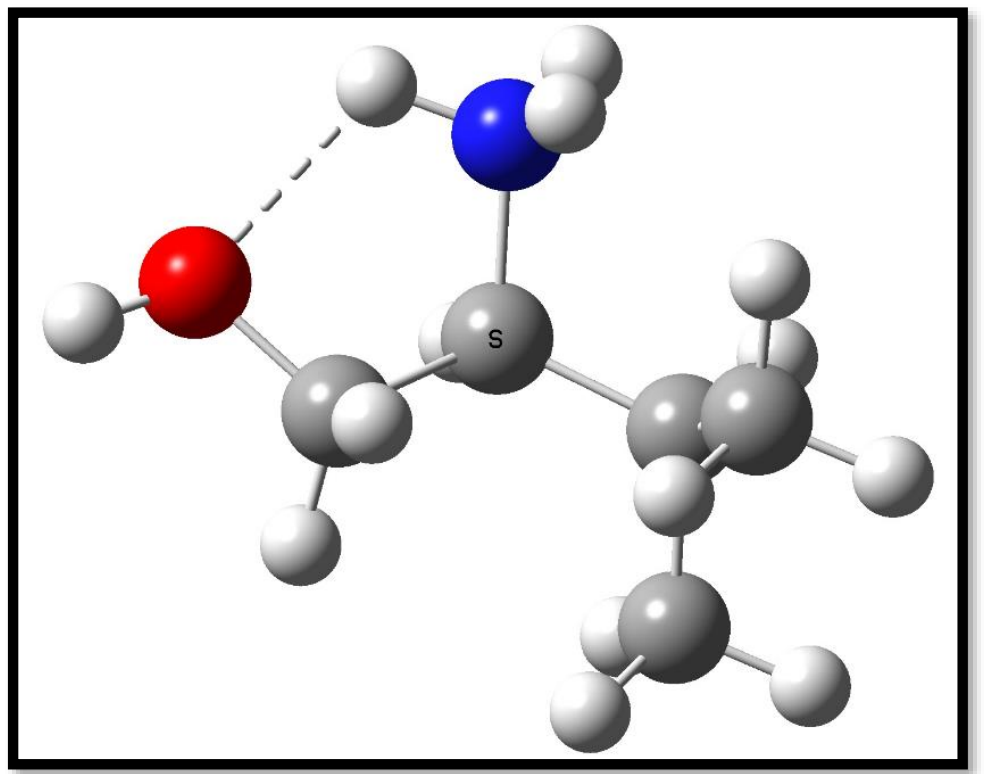

Fig. S5 Valinol with a five-membered intramolecular bond, energy $-9.2 \mathrm{kcal} / \mathrm{mol}$. Bond distance: 1.98 Angstroms. 


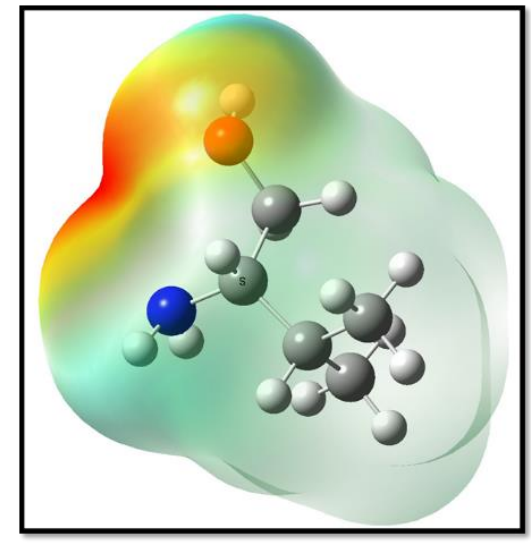

Valinol. Energy: -328.26 Hartree

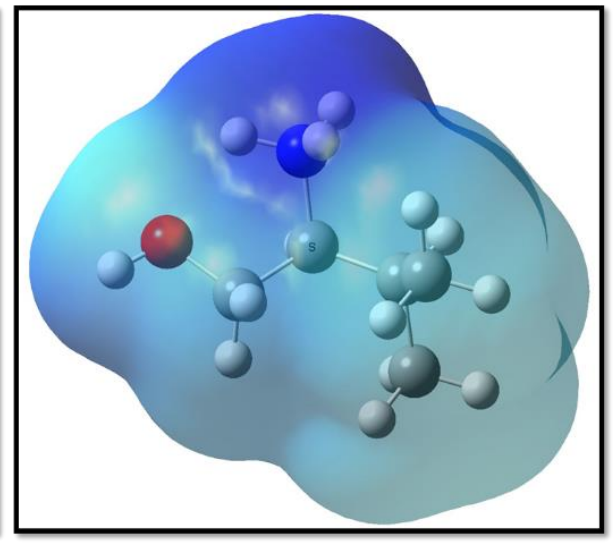

Valinol protonated on the nitrogen Energy: -328.65 Hartree IHB: $9.22 \mathrm{kcal} / \mathrm{mol}$

PA: $238.67 \mathrm{kcal} / \mathrm{mol}$

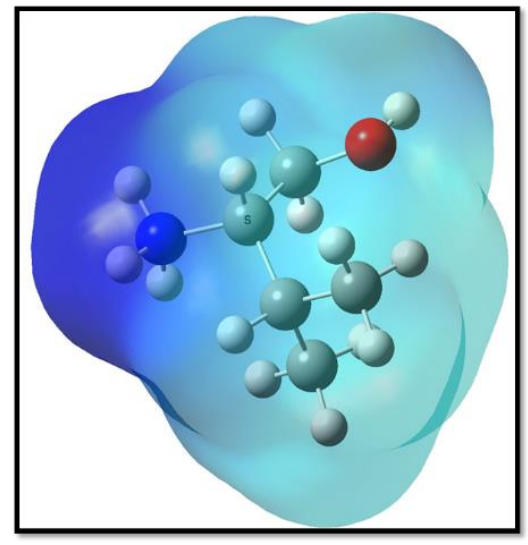

Valinol protonated on the nitrogen (anti posición) Energy: -328.63 Hartree PA: $229.45 \mathrm{kcal} / \mathrm{mol}$

Fig. S6 The potential energy surface map shows that when valinol protonation takes place an intramolecular bond of $9.22 \mathrm{kcal} / \mathrm{mol}$ is formed. Upon protonation of valinol, we can notice the change of the electrophilic zone of oxygen and nitrogen from reddish-yellow to dark blue denoting a nucleophilic zone that includes the intramolecular bond. If we force the anti position between the amino and hydroxyl group when protonation takes place, we can observe a small difference in the stabilization energy of the molecule of approximately 0.02 Hartree indicating that the structure with the highest stabilization energy and forming the intramolecular bond is the most favored. PA: proton affinity. IHB: intramolecular hydrogen bond. 


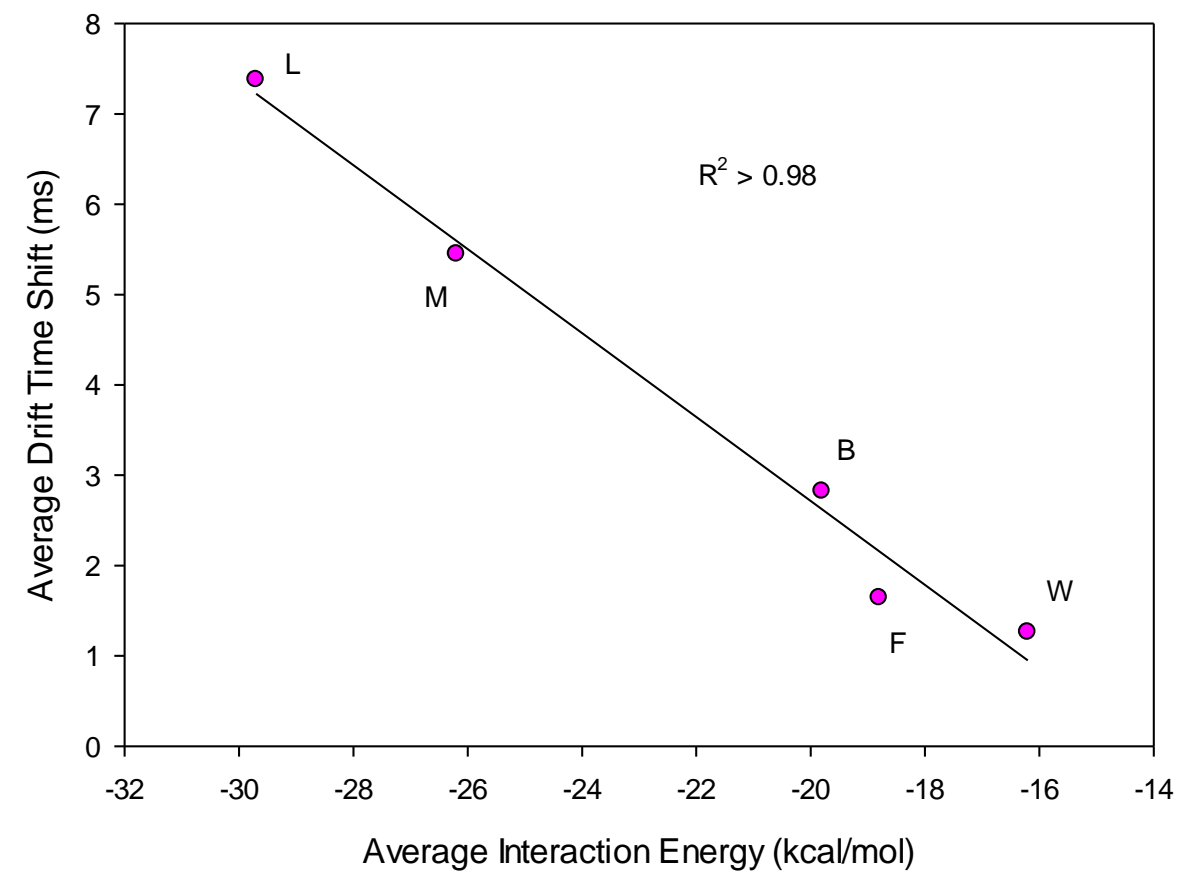

Fig. S7 The average shifts in drift time versus the average interaction energy for different SR-ion clusters, using data in Tables 2 and 3. 
Table S1. Mobility shifts, $\% \Delta \mathrm{K}_{0}$, for selected ions when different SR were introduced into the buffer gas at different concentrations in $\mathrm{mmol} \mathrm{m} \mathrm{m}^{-3}$. Ions here were not considered in Table 1 because they form intramolecular bonds or present steric hindrance on the positive charge that deter SR adduction. $\Delta \mathrm{K} 0$ values were calculated as the percent difference mobilities in nitrogen-only buffer gas and SR-doped buffer gas. The concentrations of SR in the buffer gas were 2.3, 1.0, 879, 1.7, 6.8, and $1.0 \mathrm{mmol} \mathrm{m}-3$, for F, M, W, L, B, and $\mathrm{N}$, respectively (Fernandez-Maestre et al. 2010a, 2010b).

\begin{tabular}{|c|c|c|c|c|c|c|c|c|}
\hline Compound & Mass & $\mathbf{M}$ & $\mathbf{F}$ & $\mathbf{L}$ & $\mathbf{N}$ & $\mathbf{B}$ & $\mathbf{n}$ & $\mathbf{W}$ \\
\hline SR concentration $\rightarrow$ & & & 2.3 & & 1.0 & 6.8 & $22 *$ & 879 \\
\hline SR mass $\rightarrow$ & & 122.2 & 176.1 & 118.1 & 123.1 & 74.1 & 34 & 18 \\
\hline 2,4-lutidine & 107.1 & -19 & -1.2 & -24 & -12 & -5.6 & -0.3 & -6.9 \\
\hline RIPs & - & - & - & - & - & - & -1.9 & -3.8 \\
\hline DTBP & 191.3 & -0.9 & -1.3 & -1.5 & -0.4 & -2.2 & 0.0 & -2.1 \\
\hline TBA & 242.5 & -0.1 & 0.0 & -0.3 & 0.0 & -0.3 & -0.4 & -0.9 \\
\hline TEA & 130.3 & -0.1 & 0.0 & -0.5 & 0.0 & -1.0 & -0.5 & -2.9 \\
\hline TMA & 74.2 & -0.4 & 0.0 & -1.0 & -1.0 & -1.0 & -0.3 & -4.0 \\
\hline TPA & 186.4 & -0.1 & 0.0 & -0.1 & 0.0 & 0.0 & -0.2 & -1.8 \\
\hline Methionine & 149.2 & -14 & -4.7 & - & - & - & - & -5.5 \\
\hline Phenylalanine & 165.2 & -21 & -7.3 & - & - & - & - & -5.1 \\
\hline Asparagine & 132.1 & - & - & - & - & - & - & -7.9 \\
\hline Serine & 105.1 & -29 & -10.6 & -30 & - & -14 & -4.8 & - \\
\hline Threonine & 119.1 & -23 & -8.6 & - & - & - & - & -6.6 \\
\hline Tyrosine & 181.2 & -16 & -7.0 & - & - & - & - & -5.1 \\
\hline Tryptophan & 204.2 & -10 & -4.0 & - & - & - & - & -2.2 \\
\hline Tribenzylamine & 287.4 & -6.1 & -3.1 & - & - & - & -0.3 & -1.0 \\
\hline Tributylamine & 185.3 & -15 & -6.2 & - & - & - & - & -2.0 \\
\hline Caffeine & 194.2 & - & - & -3.2 & - & -1.7 & - & - \\
\hline Glucosamine & 179.2 & - & - & -6.9 & - & -2.4 & - & - \\
\hline Valinol & $\mathbf{1 0 3 . 2}$ & $\mathbf{- 1 8}$ & $\mathbf{- 5 . 1}$ & $\mathbf{- 2 8}$ & $\mathbf{- 2 2}$ & $\mathbf{- 9 . 8}$ & $\mathbf{- 0 . 4}$ & $\mathbf{- 7 . 1}$ \\
\hline Desipramine & 266.4 & & & -12 & & -1.1 & - & - \\
\hline Atenolol & 266.4 & -0.1 & 0.1 & -7.0 & -1.6 & -0.7 & 0.0 & - \\
\hline Ethanolamine & 61.1 & -68 & -13 & -41 & -61 & -19 & - & -9.2 \\
\hline
\end{tabular}

* The water in the ammonia solution, $106 \mathrm{mmol} / \mathrm{m}^{3}$ of water, also affects the mobility. A: 2-methyl-3pentanamine, B: 2-butanol, F: trifluoromethyl benzyl alcohol, L: ethyl lactate, M: methyl-2-chloro propionate, $\mathrm{N}$ : nitrobenzene, $\mathrm{n}$ : ammonia, $\mathrm{W}$ : water.

(Fernandez-Maestre 2018). 
Table S2. Interaction energies (IE) and proton affinities (PA) for the compounds and adducts investigated. Calculations were made using Gaussian 09 (Revision D.01) at $150^{\circ} \mathrm{C}$ and the X3LYP-GD3/6-311++(d,p) functional. The data for the same modifiers are highlighted. The clusters are ordered by increasing mass.

\begin{tabular}{|c|c|c|c|c|c|}
\hline Species & PA kcal/mol & PA kJ/mol & Species & IE kcal $/ \mathrm{mol}$ & IE $\mathrm{kJ} / \mathrm{mol}$ \\
\hline $\mathrm{DH}^{+}$ & -234.73 & -982.09 & B-A & -17.95 & -75.13 \\
\hline $\mathrm{EtH}^{+}$ & -229.52 & -960.30 & F-A & -17.78 & -74.37 \\
\hline $\mathrm{PH}^{+}$ & -225.13 & -941.96 & L-A & -26.11 & -109.26 \\
\hline $\mathrm{SH}^{+}$ & -217.81 & -911.33 & M-A & -22.45 & -93.93 \\
\hline $\mathrm{ThrH}^{+}$ & -229.05 & -958.33 & $\mathrm{~N}-\mathrm{A}$ & -21.69 & -90.76 \\
\hline TryH $^{+}$ & -228.04 & -954.14 & W-A & -15.22 & -63.68 \\
\hline $\mathrm{TyrH}^{+}$ & -224.79 & -940.52 & & & \\
\hline $\mathrm{VH}^{+}$ & -238.67 & -998.60 & & & \\
\hline $\mathrm{AH}^{+}$ & -232.29 & -971.90 & & & \\
\hline
\end{tabular}

\begin{tabular}{|c|c|c|}
\hline Species & IE kcal/mol & IE kJ/mol \\
\hline B-Et & -20.22 & -84.60 \\
\hline B-V & -17.07 & -71.40 \\
\hline B-S & -23.92 & -100.10 \\
\hline B-Thr & -19.57 & -81.90 \\
\hline B-P & -20.72 & -86.70 \\
\hline B-Tyr & -20.01 & -83.70 \\
\hline B-Try & -19.57 & -81.90 \\
\hline B-D & -18.93 & -79.20 \\
\hline F-Et & -19.80 & -82.85 \\
\hline F-P & -19.41 & -81.20 \\
\hline F-S & -21.10 & -88.29 \\
\hline F-Thr & -17.58 & -73.56 \\
\hline F-Try & -18.81 & -78.71 \\
\hline F-Tyr & -19.25 & -80.56 \\
\hline F-V & -17.08 & -71.47 \\
\hline L-Et & -28.80 & -120.50 \\
\hline L-V & -25.25 & -105.63 \\
\hline L-S & -40.84 & -170.89 \\
\hline L-D & -27.82 & -116.42 \\
\hline M-Et & -25.07 & -104.92 \\
\hline M-P & -33.55 & -140.38 \\
\hline M-S & -35.44 & -148.27 \\
\hline M-Thr & -24.25 & -101.48 \\
\hline M-Try & -22.71 & -95.02 \\
\hline M-Tyr & -24.79 & -103.73 \\
\hline M-V & -21.67 & -90.66 \\
\hline W-Et & -17.18 & -71.89 \\
\hline W-P & -16.71 & -69.93 \\
\hline W-Thr & -16.51 & -69.06 \\
\hline W-Try & -16.33 & -68.32 \\
\hline W-Tyr & -17.22 & -72.04 \\
\hline W-V & -14.59 & -61.05 \\
\hline A $-\mathrm{m}$ \\
\hline
\end{tabular}

A: 2-methyl-3-pentanamine, B: 2-butanol, D: desipramine, Et: ethanolamine, F: trifluoromethyl benzyl alcohol, L: ethyl lactate, Met: methionine, M: methyl-2-chloro propionate, P: phenylalanine, Thr: threonine, Try: tryptophan, Tyr: tyrosine, S: serine, V: Valinol, W: water. 
Table S3. Interaction energy differences without zero-point vibrational energies (IE), Gibbs free energies $(\Delta \mathrm{G})$, enthalpies $(\Delta \mathrm{H})$ and entropies (reported as $\mathrm{T} \Delta \mathrm{S}$ ) for the complex formation reactions studied. Calculations were made using Gaussian 09 (Revision D.01) at $150^{\circ} \mathrm{C}$ and the X3LYP-GD3/6-311++(d,p) functional and calculation basis. All energies are given in $\mathrm{kJ} / \mathrm{mol}$. The data for the same modifiers are highlighted. The clusters are ordered by increasing mass.

\begin{tabular}{|c|c|c|c|c|c|c|c|c|c|}
\hline Species & $\begin{array}{c}\Delta \mathrm{H} \\
(\mathrm{kJ} / \mathrm{mol})\end{array}$ & $\begin{array}{c}\Delta \mathrm{G} \\
(\mathrm{kJ} / \mathrm{mol})\end{array}$ & $\begin{array}{c}\mathrm{T} \Delta \mathrm{S} \\
(\mathrm{kJ} / \mathrm{mol})\end{array}$ & $\begin{array}{c}\mathrm{IE} \\
(\mathrm{kJ} / \mathrm{mol})\end{array}$ & Species & $\begin{array}{c}\Delta \mathrm{H} \\
(\mathrm{kJ} / \mathrm{mol})\end{array}$ & $\begin{array}{c}\Delta \mathrm{G} \\
(\mathrm{kJ} / \mathrm{mol})\end{array}$ & $\begin{array}{c}\mathrm{T} \Delta \mathrm{S} \\
(\mathrm{kJ} / \mathrm{mol})\end{array}$ & $\begin{array}{c}\mathrm{IE} \\
(\mathrm{kJ} / \mathrm{mol})\end{array}$ \\
\hline B-Et & -79.6 & -41.3 & -38.3 & -84.6 & M-Et & -99.8 & -61.8 & -38.0 & -104.9 \\
\hline B-V & -103.6 & -63.2 & -40.4 & -71.4 & $M-V$ & -123.1 & -83.3 & -39.8 & -90.7 \\
\hline B-S & -94.9 & -54.5 & -40.5 & -100.1 & M-S & -142.5 & -105.7 & -36.8 & -148.3 \\
\hline B-Thr & -76.1 & -36.5 & -39.6 & -81.9 & M-Thr & -95.7 & -57.9 & -37.8 & -101.5 \\
\hline B-P & -81.3 & -43.8 & -37.5 & -86.7 & M-P & -134.6 & -98.9 & -35.7 & -140.4 \\
\hline B-Tyr & -78.0 & -42.9 & -35.0 & -83.7 & M-Tyr & -98.2 & -58.5 & -39.7 & -103.7 \\
\hline B-Try & -75.9 & -36.2 & -39.7 & -81.9 & M-Try & -89.6 & -52.7 & -36.8 & -95.0 \\
\hline B-D & -73.9 & -30.8 & -43.1 & -79.2 & & & & & \\
\hline F-Et & -76.9 & -38.4 & -38.5 & -82.9 & W-Et & -65.8 & -32.2 & -33.6 & -71.9 \\
\hline F-V & -103.2 & -60.1 & -43.1 & -71.5 & W-V & -92.9 & -60.2 & -32.7 & -61.0 \\
\hline F-S & -82.0 & -41.3 & -40.6 & -88.3 & W-Thr & -62.6 & -30.2 & -32.4 & -69.1 \\
\hline F-Thr & -67.5 & -31.5 & -36.0 & -73.6 & W-P & -63.4 & -29.7 & -33.8 & $-69,9$ \\
\hline F-P & -77.3 & -29.4 & -48.0 & -81.2 & W-Tyr & -65.7 & -32.6 & -33.1 & -72.0 \\
\hline F-Tyr & -74.0 & -34.3 & -39.7 & -80.6 & W-Try & -62.0 & -25.0 & -37.0 & -68.3 \\
\hline F-Try & -72.0 & -32.1 & -40.0 & -78.7 & & & & & \\
\hline L-Et & -115.8 & -77.7 & -38.1 & -120.5 & B-A & -69.1 & -27.2 & -41.9 & -75.1 \\
\hline L-V & -138.2 & -95.7 & -42.6 & -105.6 & F-A & -67.7 & -24.8 & -42.9 & -74.4 \\
\hline L-S & -164.4 & -124.8 & -39.6 & -170.9 & L-A & -103.5 & -61.8 & -41.8 & -109.3 \\
\hline L-Thr & -114.6 & -72.7 & -41.9 & -120.8 & M-A & -88.3 & -49.4 & -38.8 & -93.9 \\
\hline L-P & -113.5 & -76.9 & -36.6 & -118.7 & W-A & -57.4 & -23.6 & -33.8 & -63.7 \\
\hline L-Try & -109.0 & -69.5 & -39.5 & -114.5 & & & & & \\
\hline L-D & -148.3 & -104.3 & -44.0 & -116.4 & & & & & \\
\hline
\end{tabular}

A: 2-methyl-3-pentanamine, B: 2-butanol, D: desipramine, Et: ethanolamine, F: trifluoromethyl benzyl alcohol, L: ethyl lactate, Met: methionine, M: methyl-2-chloro propionate, P: phenylalanine, S: serine, Thr: threonine, Try: tryptophan, Tyr: tyrosine, V: Valinol, W: water. 


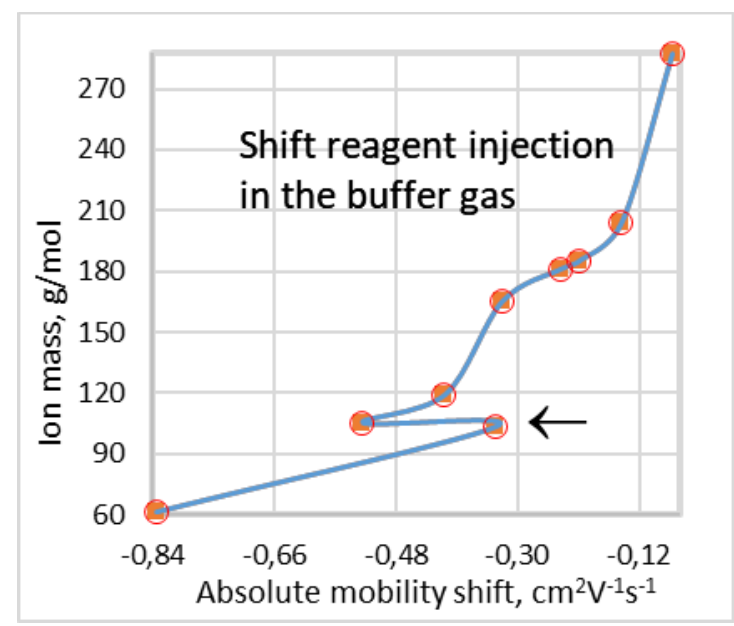

The ion:shift reagent interaction energy explains the anomalies (arrow) in the mass-mobility shift correlation in Ion Mobility Spectrometry

- Ion mobility spectrometry detects illegal substances but false positives thwart this task

- Shift reagents can eliminate false positives but this mechanism needs clarification

- Injection of shift reagents changed ion mobilities and mass-mobility shift correlation

- Ion:shift reagent interaction energies explain these changes

- False positives hinder passenger/cargo transit and seizure of illicit substances and needless delays 